\title{
Regioselective Formation of Enol Esters from the Ruthenium-catalyzed Markovnikov Addition of Carboxylic Acids to Alkynes
}

\author{
Janine Jeschke, Christian Gäbler and Heinrich Lang*
}

Technische Universität Chemnitz, Faculty of Natural Sciences, Institute of Chemistry, Inorganic Chemistry, 09107 Chemnitz, Germany.

Supporting Information 


\section{Table of Contents}

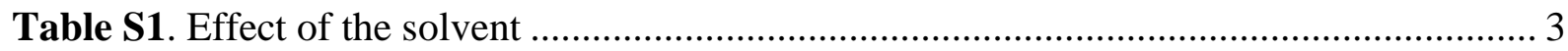

Table S2. Effect of the amount of added triflate ................................................................. 4

Figure S3. Comparison of the reaction profiles of catalysts 1a, 1c and 1e for the addition of

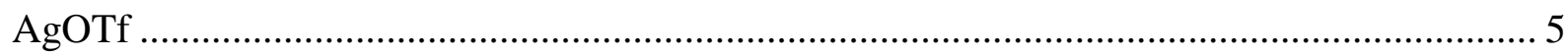

Table S4. Effect of the acid/alkyne ratio on the productivity .............................................. 6

${ }^{1} \mathrm{H}$ and ${ }^{13} \mathrm{C}\left\{{ }^{1} \mathrm{H}\right\}$ NMR Spectra of Catalysis Products 2aa-oa .......................................... 7

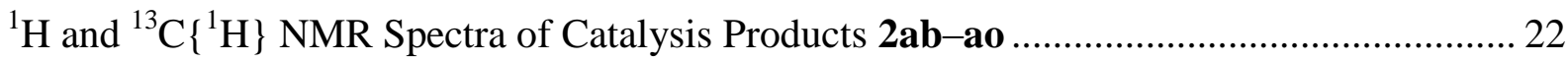

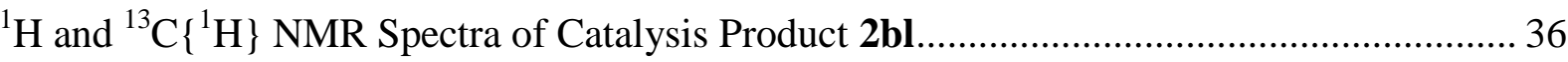


Table S1. Effect of the solvent on the activity and selectivity in the conversion of benzoic acid to phenylacetylene catalyzed by $\mathbf{1 a}{ }^{a}$

\begin{tabular}{|c|c|c|c|c|c|}
\hline \multirow{2}{*}{ Entry } & \multirow{2}{*}{ Solvent } & \multirow{2}{*}{ Yield $[\%]^{b}$} & \multicolumn{3}{|c|}{ Selectivity $[\%]^{c}$} \\
\hline & & & $2 \mathbf{a a}$ & $(Z)-\mathbf{3 a a}$ & $(E)-\mathbf{3 a a}$ \\
\hline 1 & $\mathrm{CCl}_{4}$ & 95 & 74 & 19 & 7 \\
\hline 2 & Toluene/cyclohexane $(1: 1)$ & 90 & 79 & 15 & 6 \\
\hline 3 & Toluene & 100 & 74 & 20 & 6 \\
\hline 4 & Toluene/THF (1:1) & 64 & 64 & 25 & 11 \\
\hline 5 & DCM & 83 & 66 & 28 & 6 \\
\hline 6 & THF & 53 & 42 & 43 & 15 \\
\hline 7 & Dioxane & 63 & 76 & 18 & 6 \\
\hline
\end{tabular}

${ }^{a}$ Reaction conditions: benzoic acid $(1.0 \mathrm{mmol})$, phenylacetylene $(2.0 \mathrm{mmol})$, acenaphthene (0.5 mmol), 1a (1.0 mol\%), solvent $(1 \mathrm{~mL}), 60{ }^{\circ} \mathrm{C}, 24 \mathrm{~h} .{ }^{b}$ Total yield determined by ${ }^{1} \mathrm{H}$ NMR spectroscopy applying acenaphthene as internal standard. ${ }^{c}$ Relative ratio of isomers determined by ${ }^{\mathrm{l}} \mathrm{H}$ NMR spectroscopy. 
Table S2. Effect of the amount of KOTf, $\mathrm{AgOTf}, \mathrm{Mg}(\mathrm{OTf})_{2}$ and $\mathrm{Yb}(\mathrm{OTf})_{3}$ on the productivity in the conversion of benzoic acid to phenylacetylene to give 2aa catalyzed by 1a. ${ }^{a}$

\begin{tabular}{cccccc}
\hline \multirow{2}{*}{ Entry } & Additive $[\mathrm{mol} \%]$ & \multicolumn{5}{c}{${\text { Yield }[\%]^{b}}^{b}$} & KOTf & AgOTf & $\operatorname{Mg}(\mathrm{OTf})_{2}$ & Yb(OTf) \\
\cline { 3 - 5 } & 0 & 29 & 29 & 29 & 29 \\
2 & 0.25 & 32 & 74 & 39 & 92 \\
3 & 0.5 & 35 & 88 & 41 & 72 \\
4 & 1 & 41 & 99 & 39 & 54 \\
5 & 2 & 58 & 66 & 39 & 30 \\
6 & 4 & 77 & 25 & 39 & 18 \\
\hline
\end{tabular}

${ }^{a}$ Reaction conditions: benzoic acid $(1.0 \mathrm{mmol})$, phenylacetylene $(2.0 \mathrm{mmol})$, acenaphthene $(0.5 \mathrm{mmol}), \mathbf{1 a}(1.0 \mathrm{~mol} \%)$, additive, toluene $(1 \mathrm{~mL}), 70{ }^{\circ} \mathrm{C}, 2 \mathrm{~h} .{ }^{b}$ Yield of 2 aa determined by ${ }^{1} \mathrm{H}$ NMR spectroscopy applying acenaphthene as internal standard. 


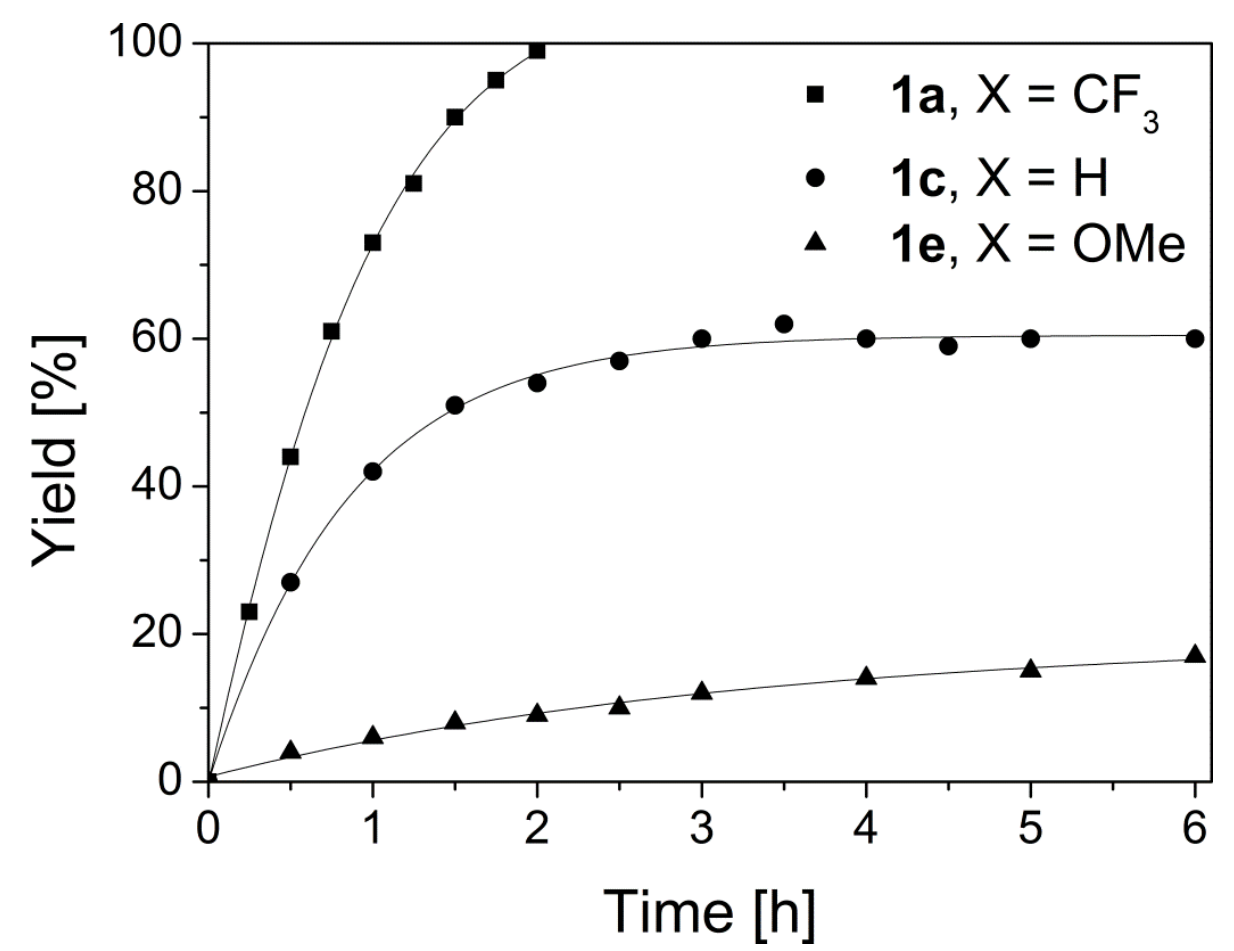

Figure S3. Reaction profiles for the addition of $1.0 \mathrm{~mol} \%$ AgOTf in the conversion of benzoic acid to phenylacetylene to give 2 aa catalyzed by $1.0 \mathrm{~mol} \%$ of $\left[\mathrm{Ru}(\mathrm{CO})_{2}\left(\mathrm{P}\left(p-\mathrm{C}_{6} \mathrm{H}_{4}-\right.\right.\right.$ $\left.\left.\mathrm{X})_{3}\right)_{2}\left(\mathrm{O}_{2} \mathrm{CPh}\right)_{2}\right]\left(\mathbf{1 a}, \mathrm{X}=\mathrm{CF}_{3} ; \mathbf{1 c}, \mathrm{X}=\mathrm{H} ; \mathbf{1 e}, \mathrm{X}=\mathrm{OMe}\right)$ at $70{ }^{\circ} \mathrm{C}$ in toluene. 
Table S4. Effect of the acid/alkyne ratio on the productivity in the conversion of benzoic acid to phenylacetylene to give $\mathbf{2 a a}$ catalyzed by $\mathbf{1 a}{ }^{a}$

\begin{tabular}{ccc}
\hline Entry & Equivalents of Alkyne & ${\text { Yield }[\%]^{b}}^{b}$ \\
\hline 1 & 1.1 & 92 \\
2 & 1.5 & 98 \\
3 & 2.0 & 99 \\
\hline
\end{tabular}

${ }^{a}$ Reaction conditions: benzoic acid $(1.0 \mathrm{mmol})$, phenylacetylene $(1.1-2.0 \mathrm{mmol})$, acenaphthene $(0.5 \mathrm{mmol}), \mathbf{1 a}(1.0 \mathrm{~mol} \%)$, AgOTf $(1.0 \mathrm{~mol} \%)$, toluene $(1 \mathrm{~mL}), 70{ }^{\circ} \mathrm{C}, 2 \mathrm{~h} .{ }^{b}$ Yield of 2aa determined by ${ }^{1} \mathrm{H}$ NMR spectroscopy applying acenaphthene as internal standard. 
${ }^{1} \mathrm{H}$ and ${ }^{13} \mathrm{C}\left\{{ }^{1} \mathrm{H}\right\}$ NMR Spectra of Catalysis Products $2 \mathrm{aa}-\mathrm{oa}$ in $\mathrm{CDCl}_{3}$
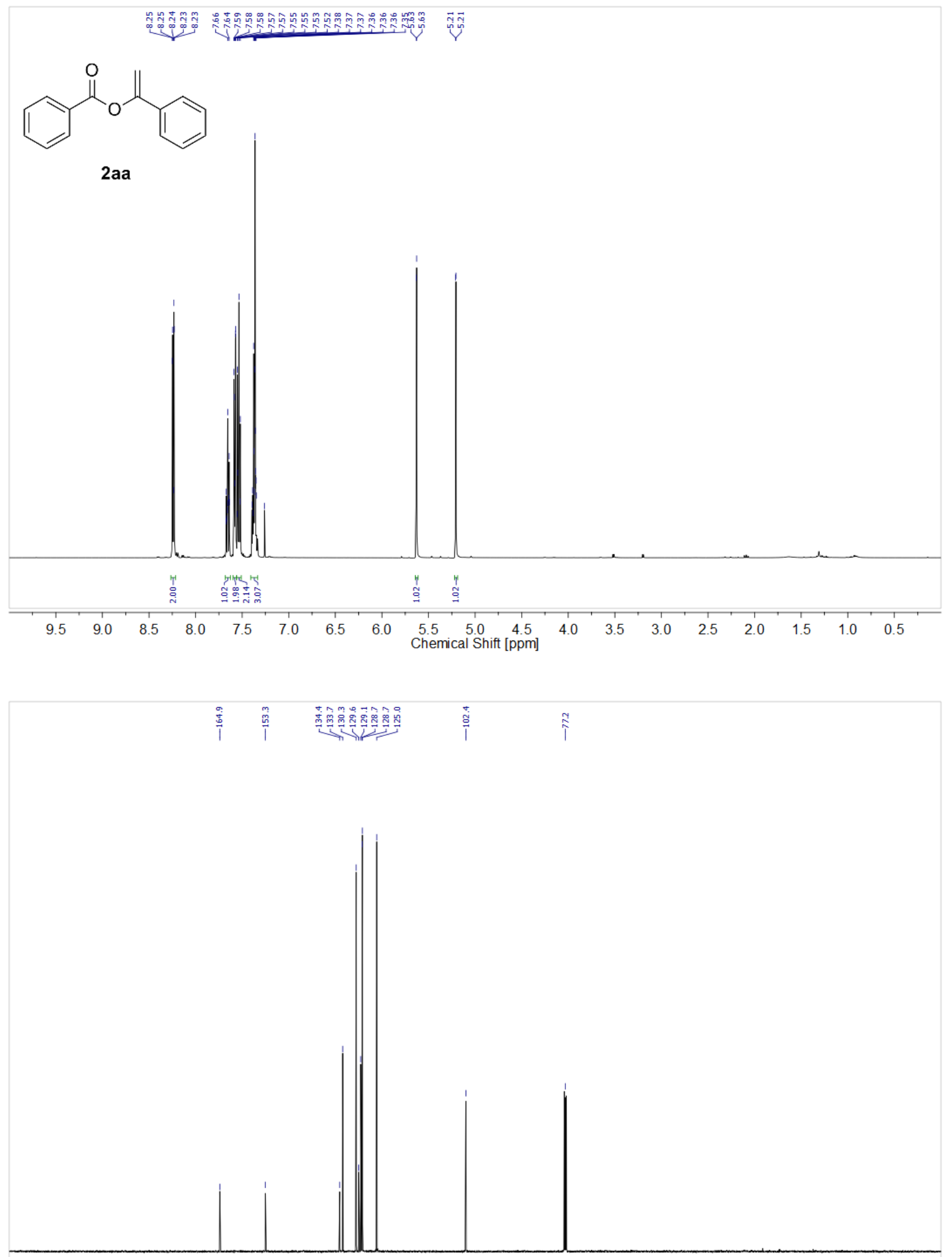

$\begin{array}{llllllllllllllllllllllll}210 & 200 & 190 & 180 & 170 & 160 & 150 & 140 & 130 & 120 & 110 & 100 & 90 & 80 & 70 & 60 & 50 & 40 & 30 & 20 & 10 & 0 & -10\end{array}$ 

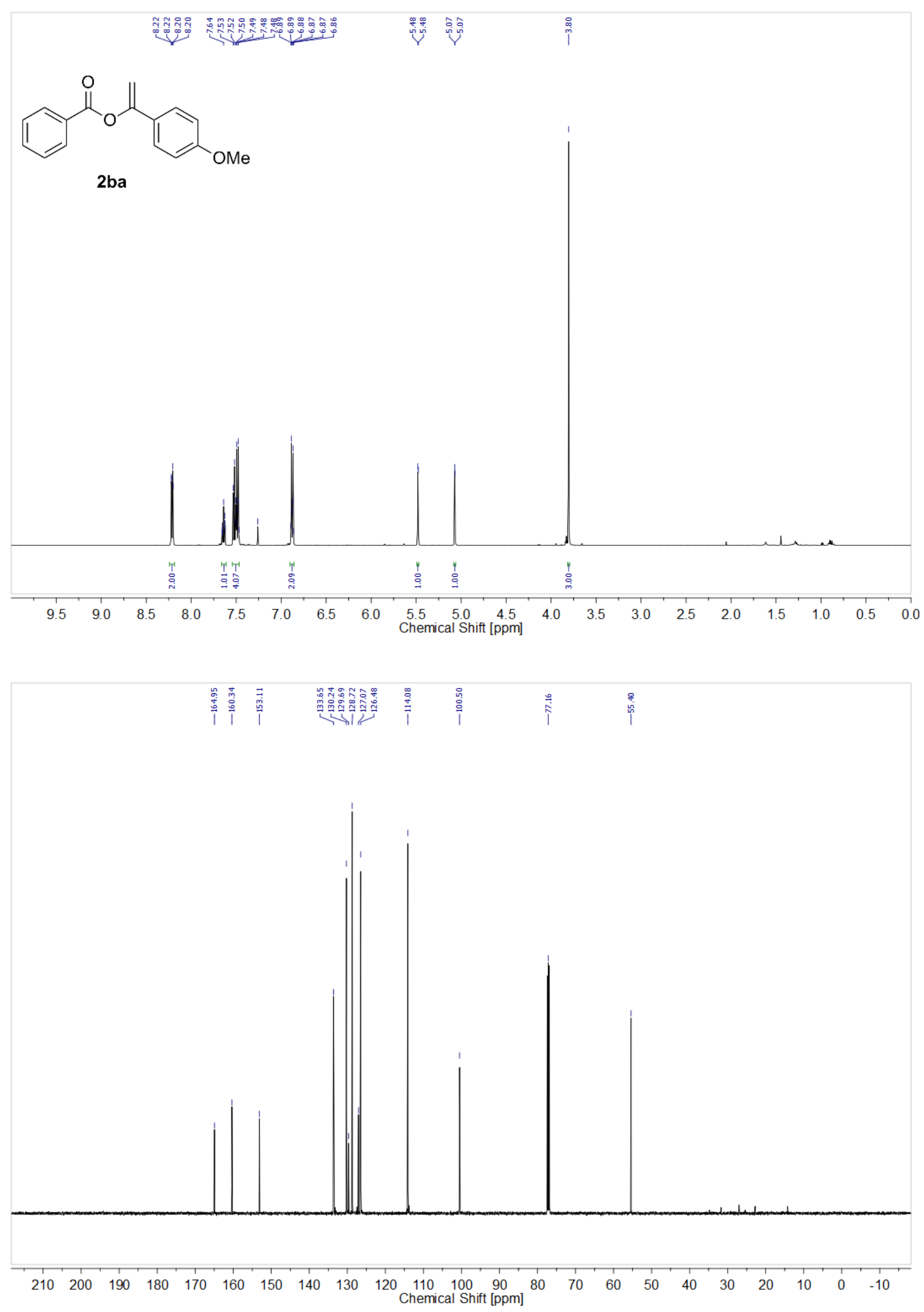

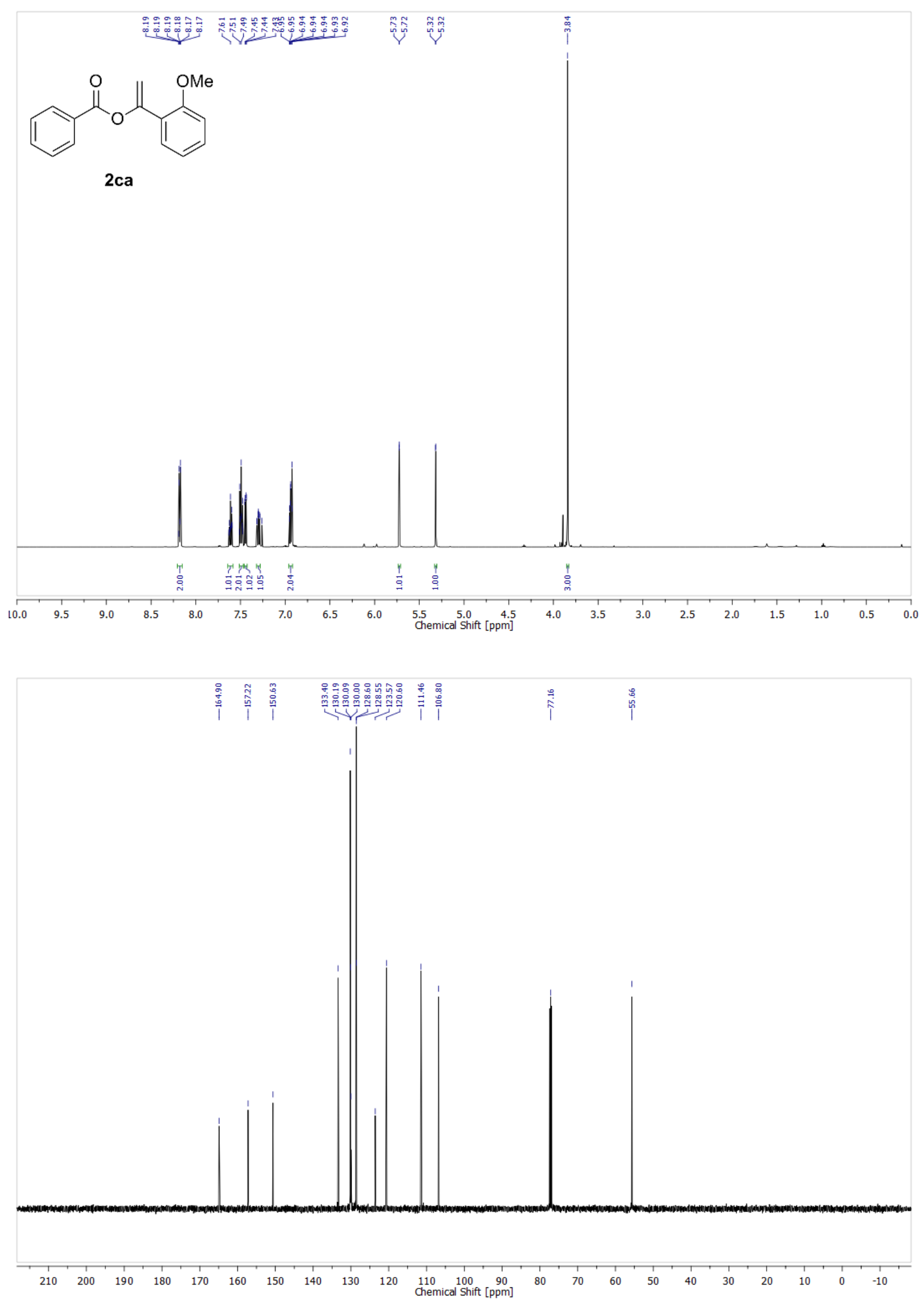

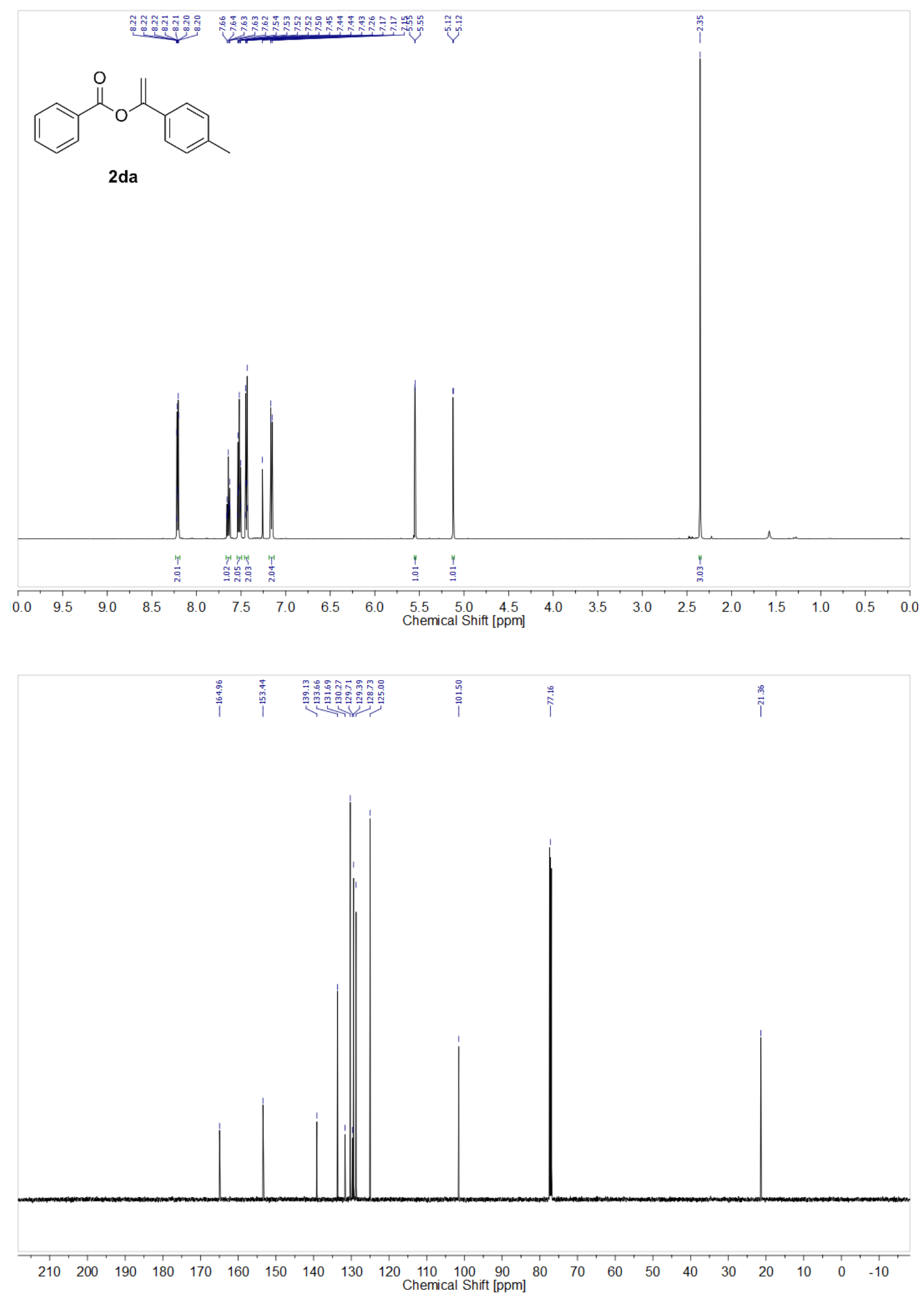

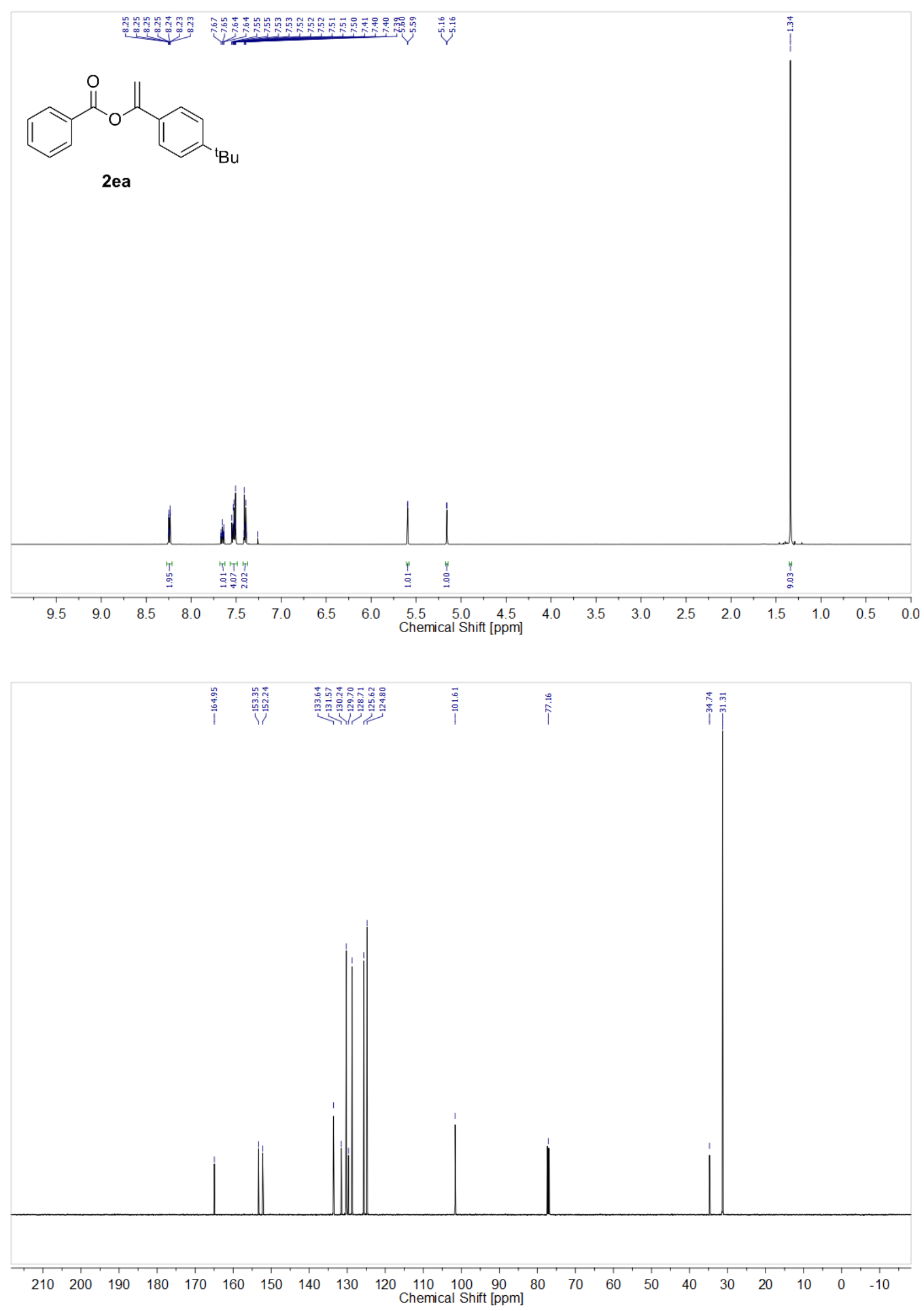

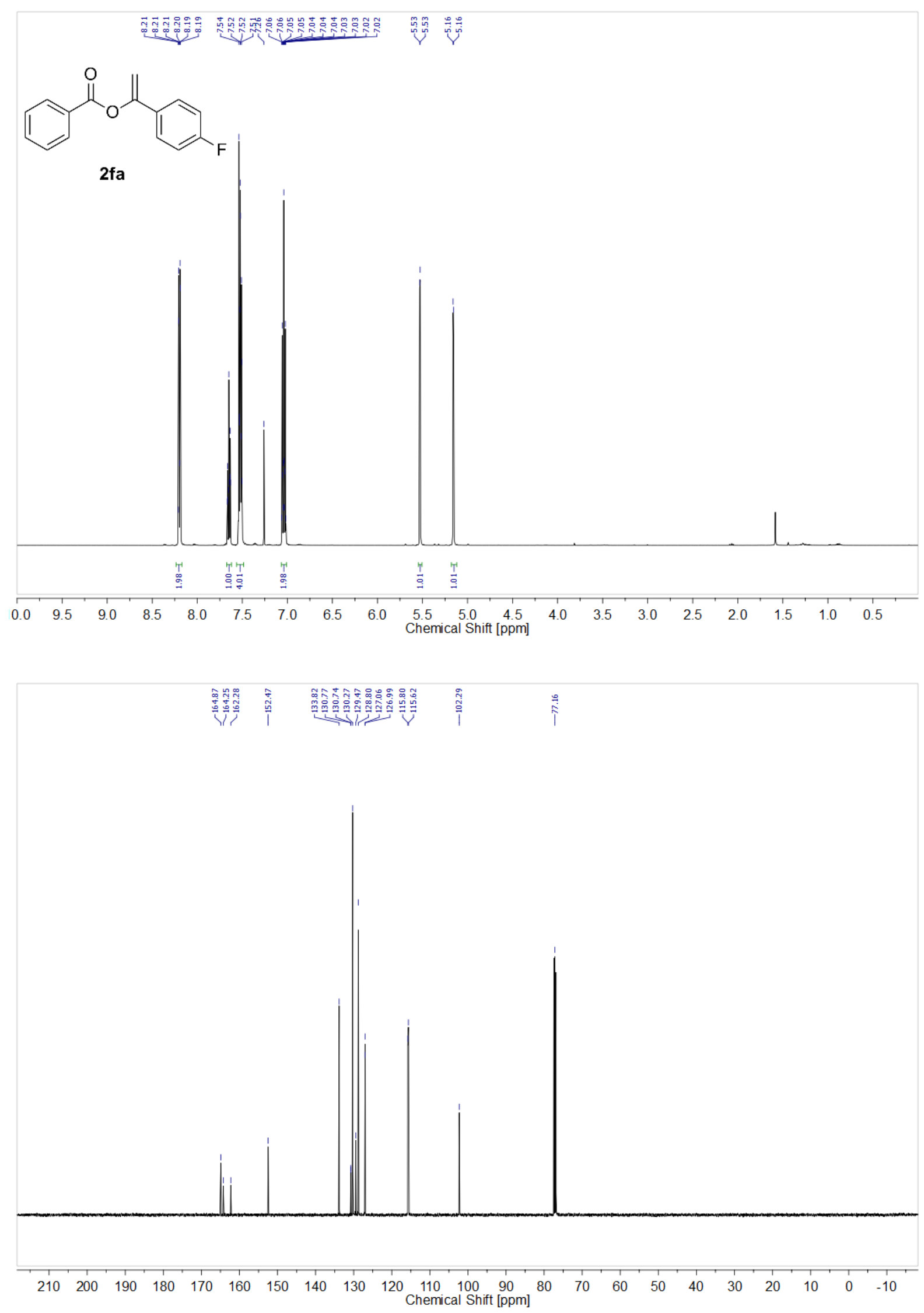

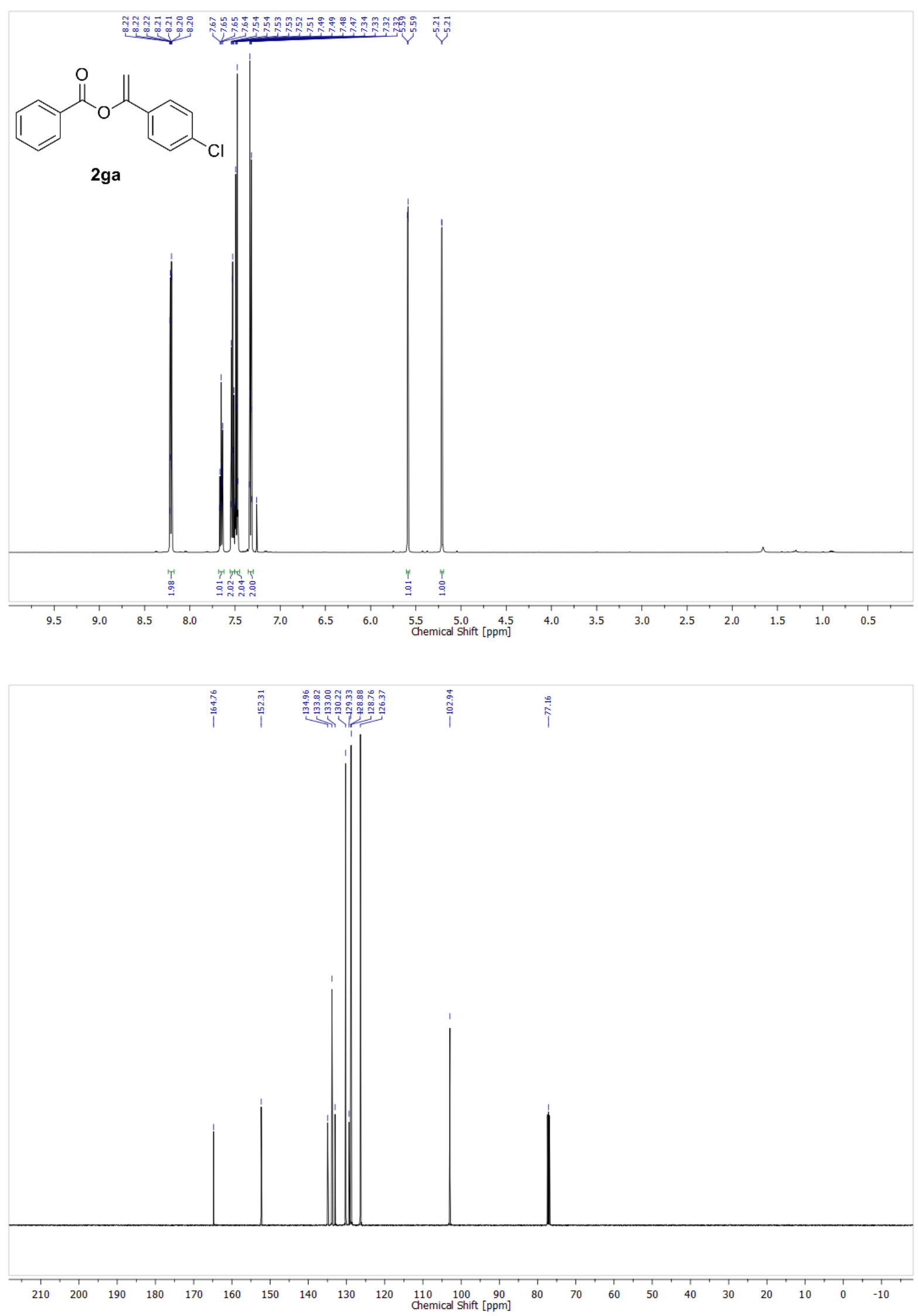

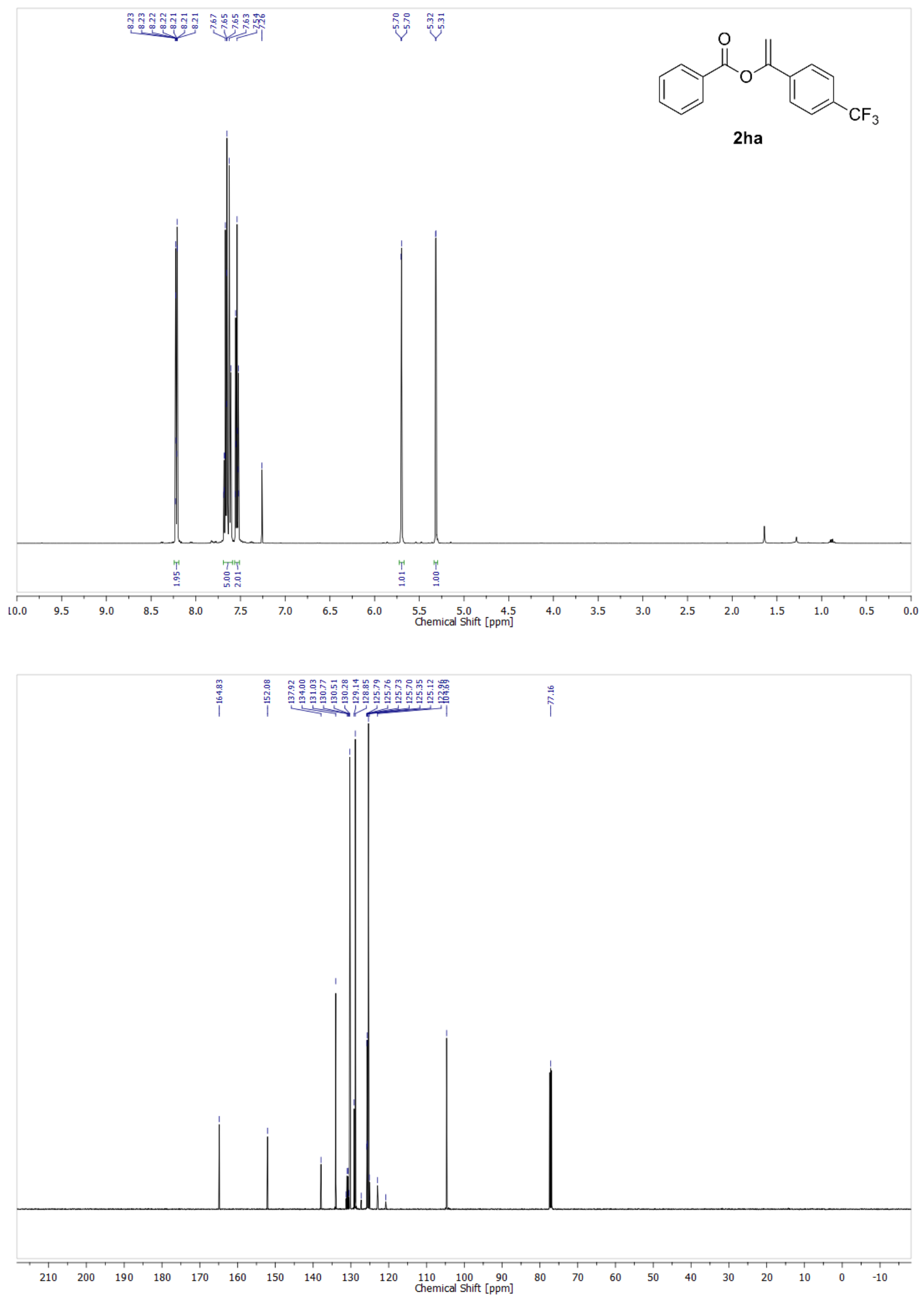

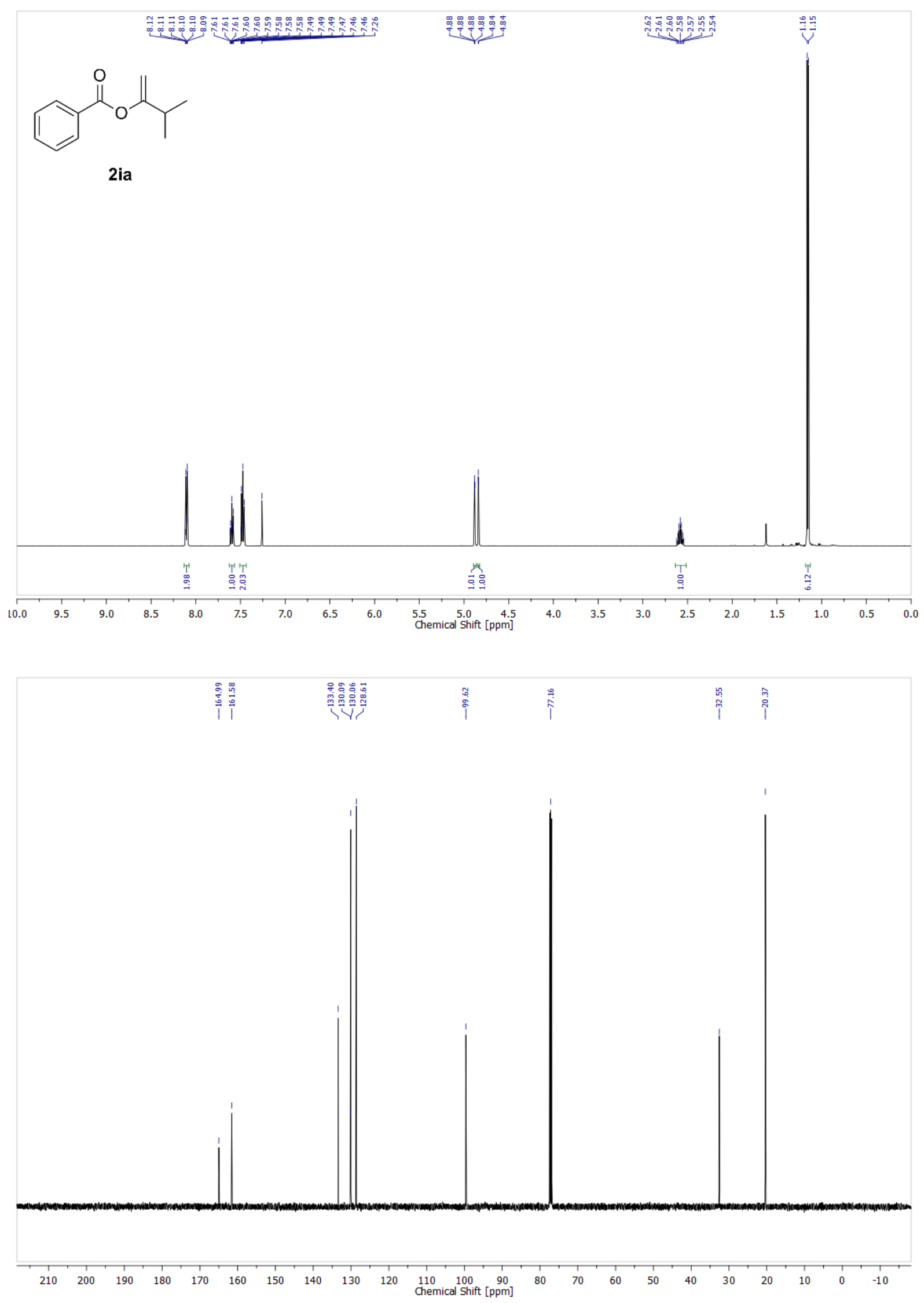

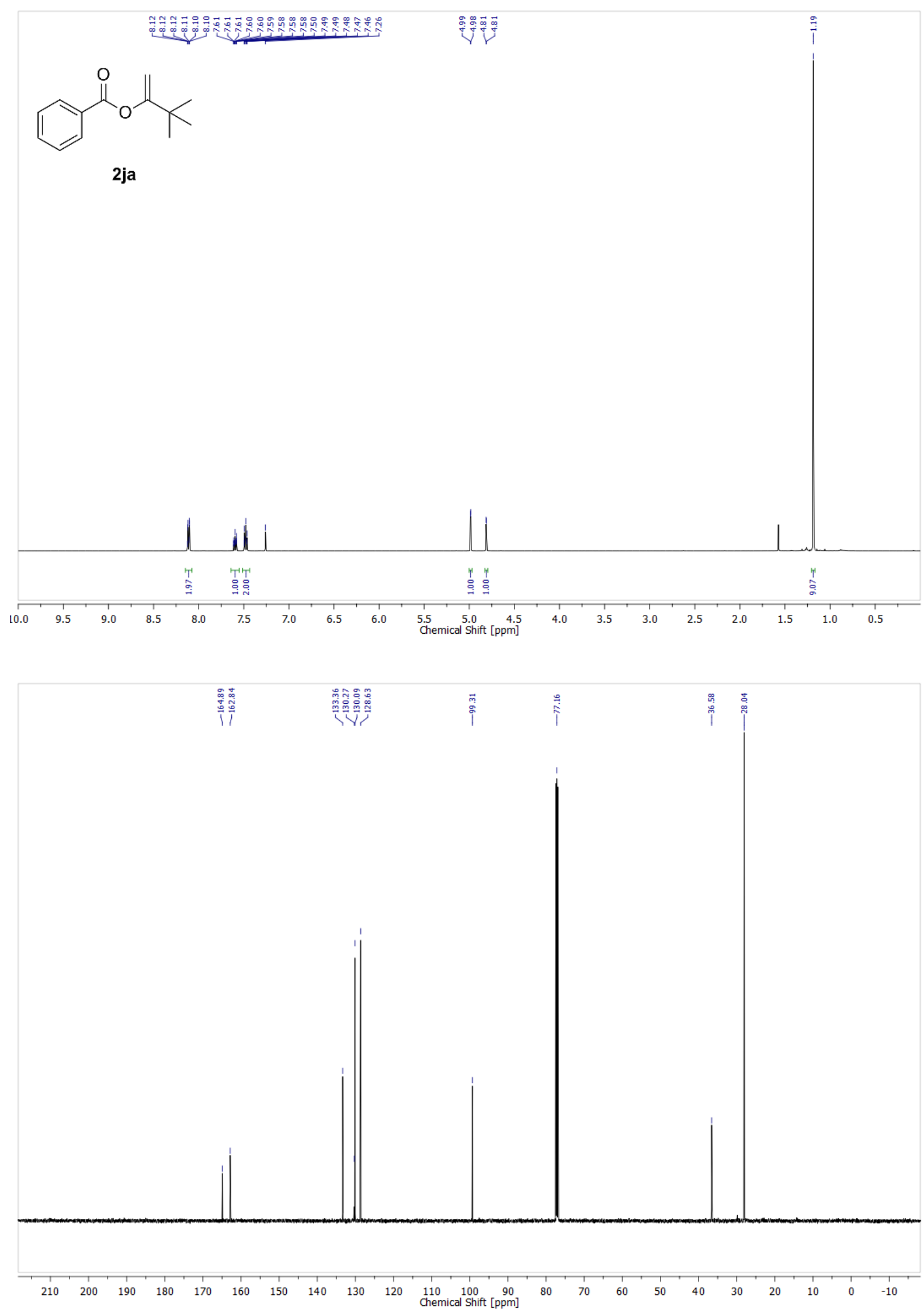

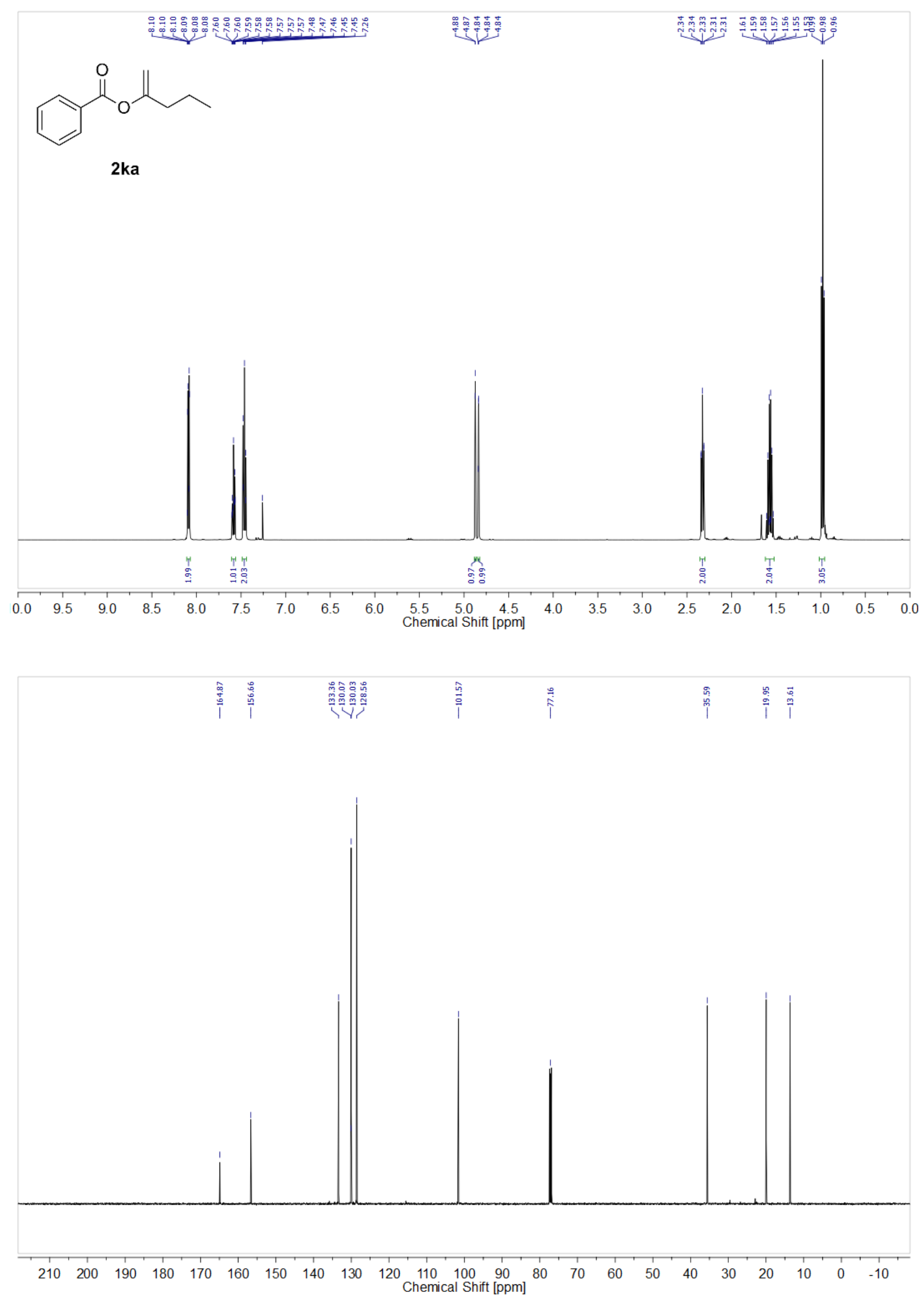

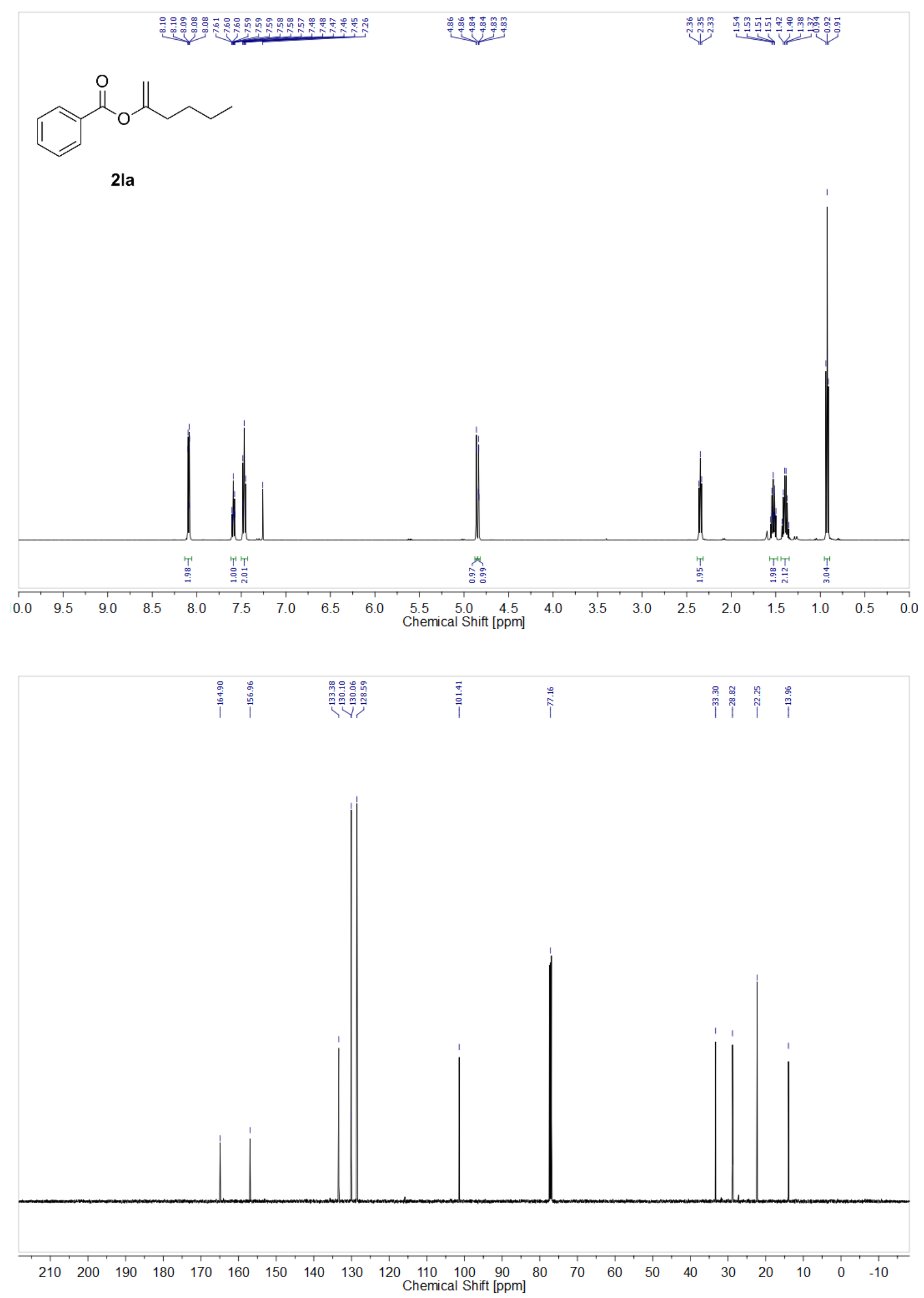

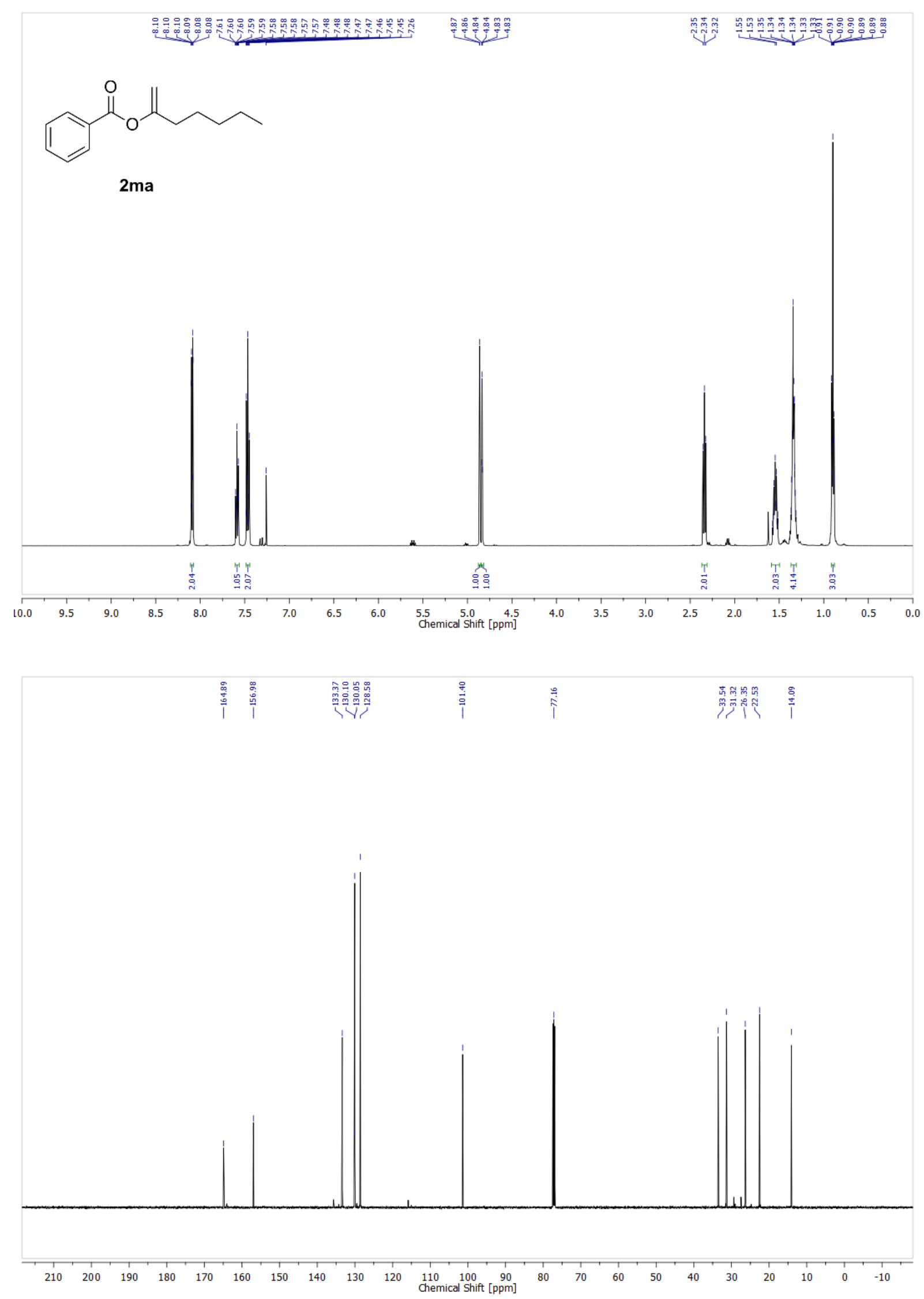

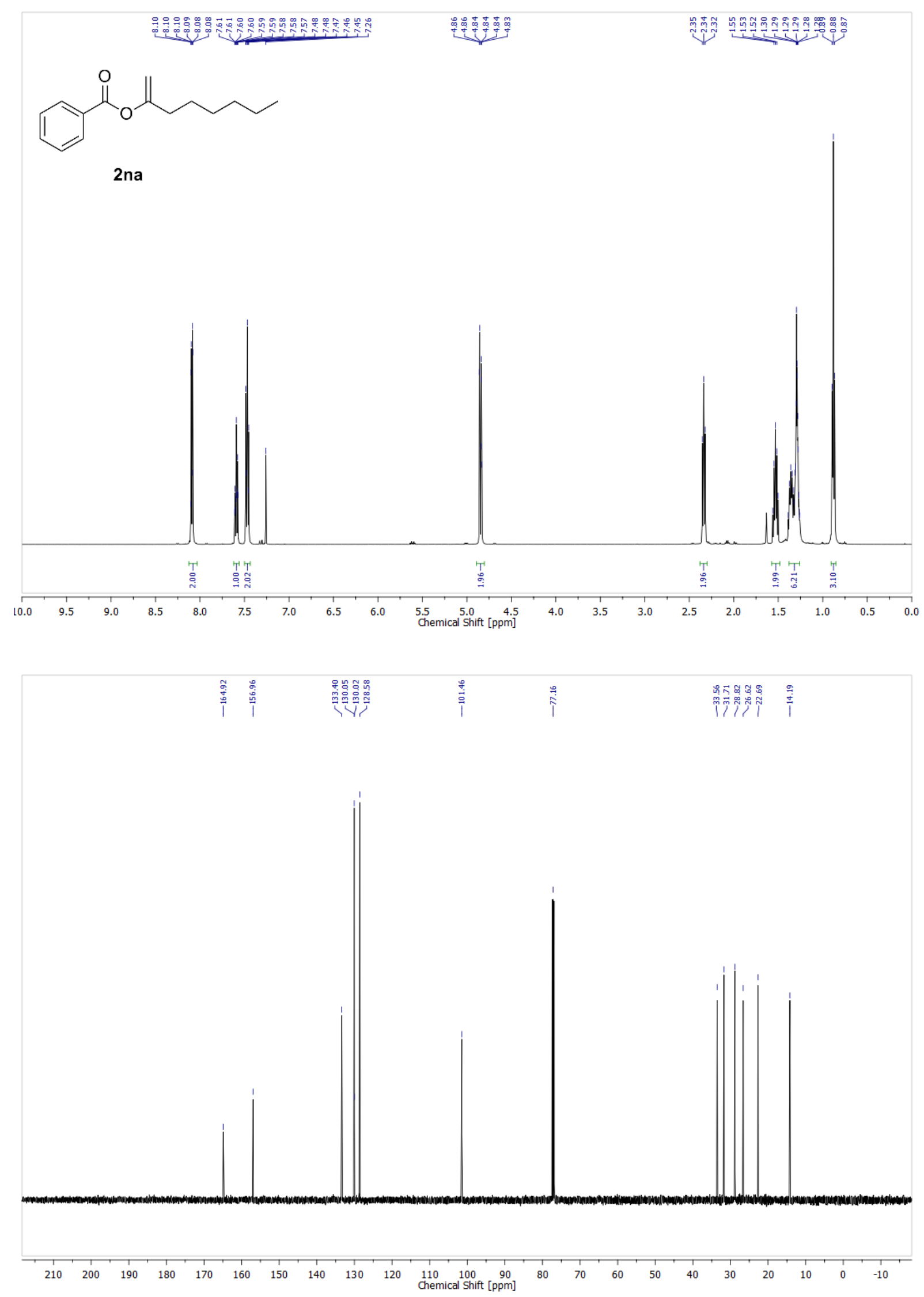

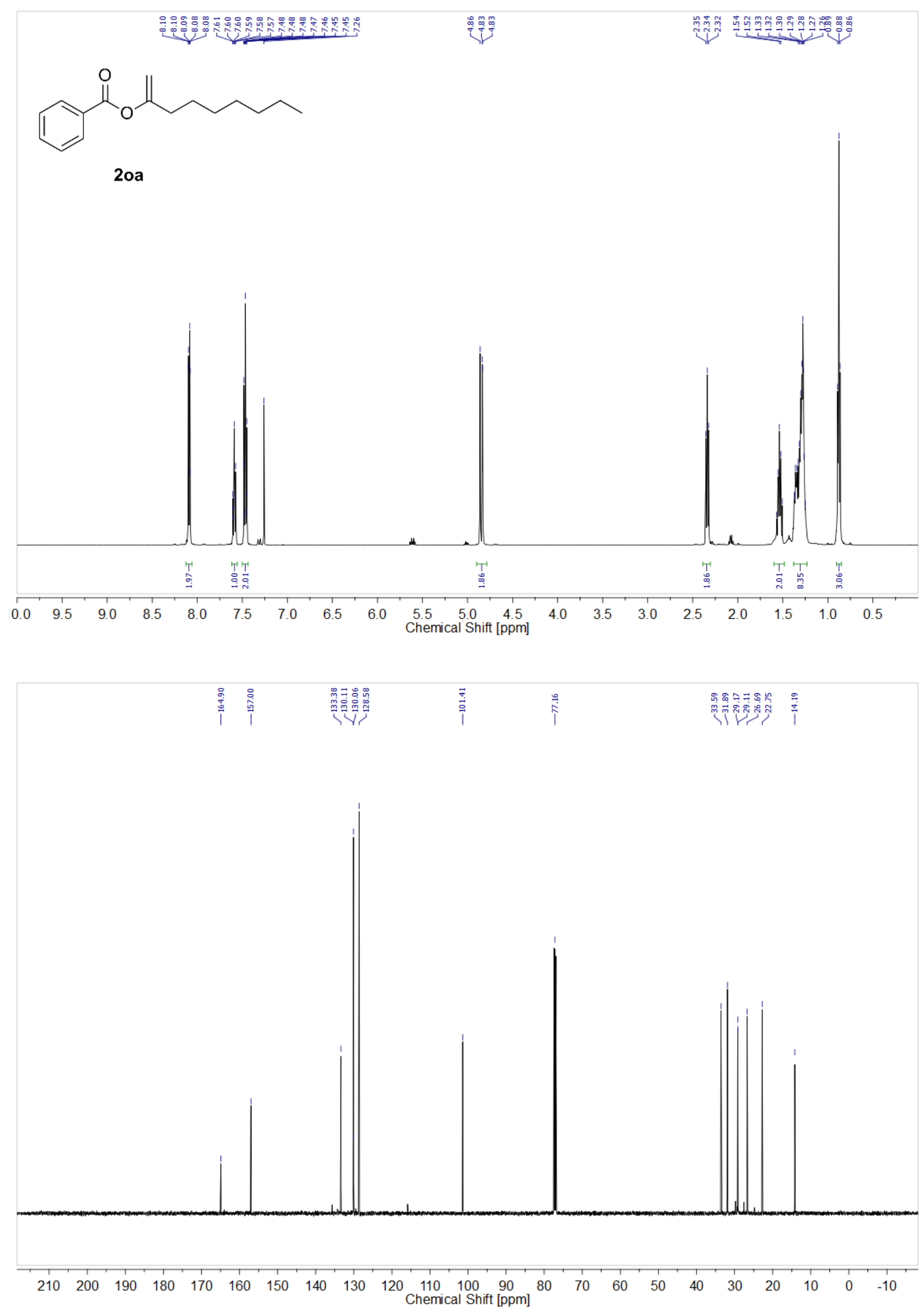
${ }^{1} \mathrm{H}$ and ${ }^{13} \mathrm{C}\left\{{ }^{1} \mathrm{H}\right\}$ NMR Spectra of Catalysis Products $2 \mathrm{ab}-\mathrm{ao}$ in $\mathrm{CDCl}_{3}$
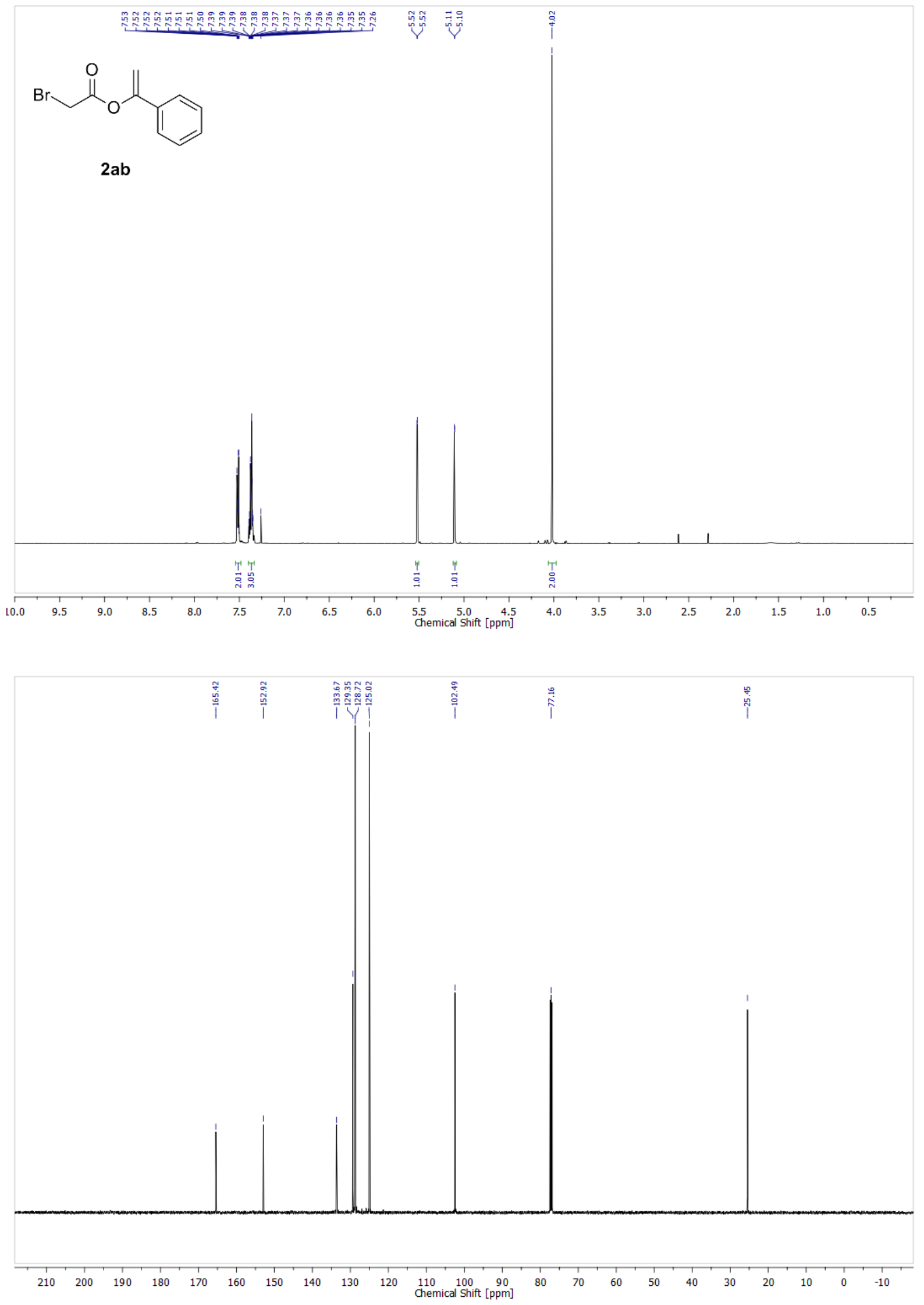

22 

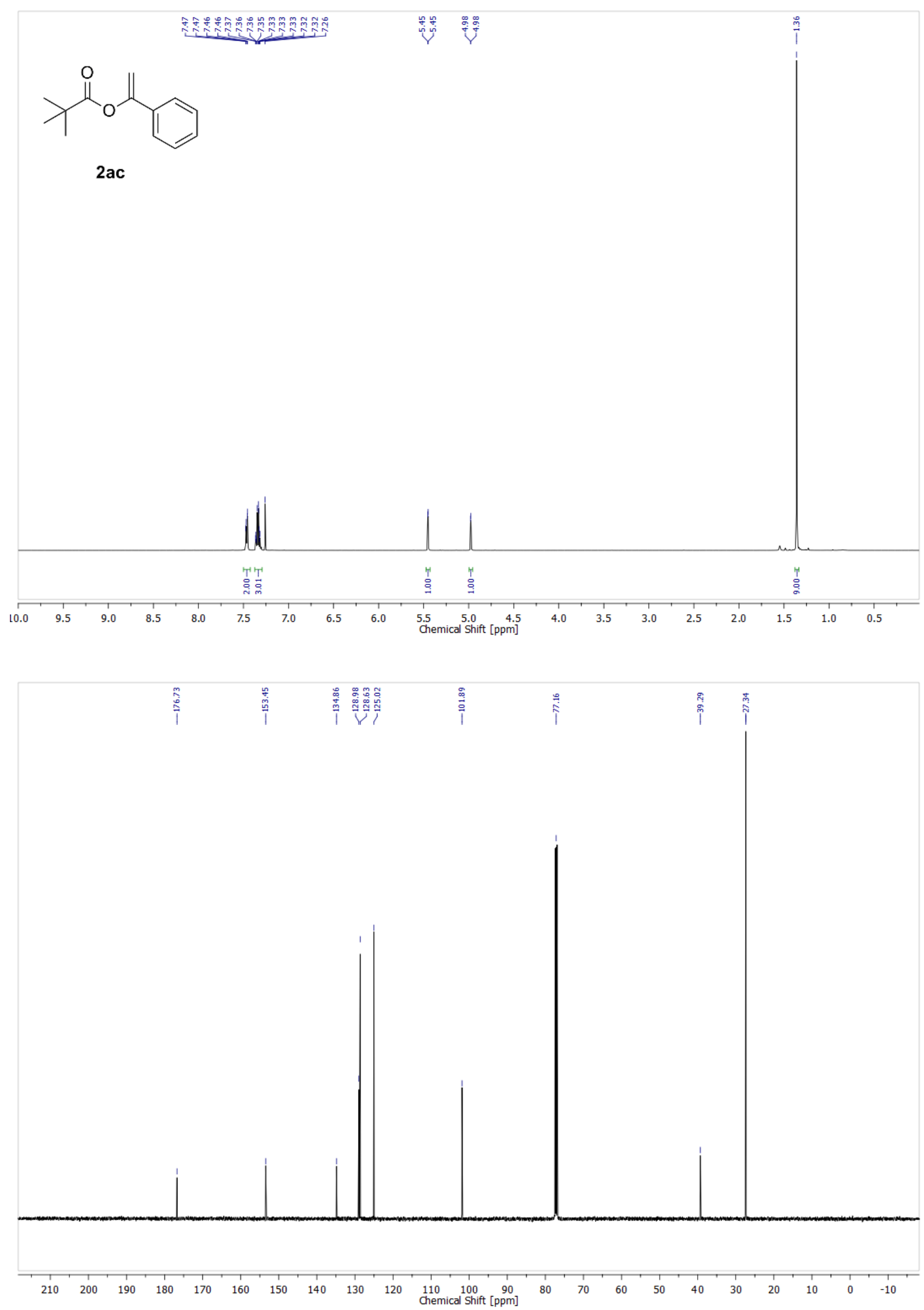

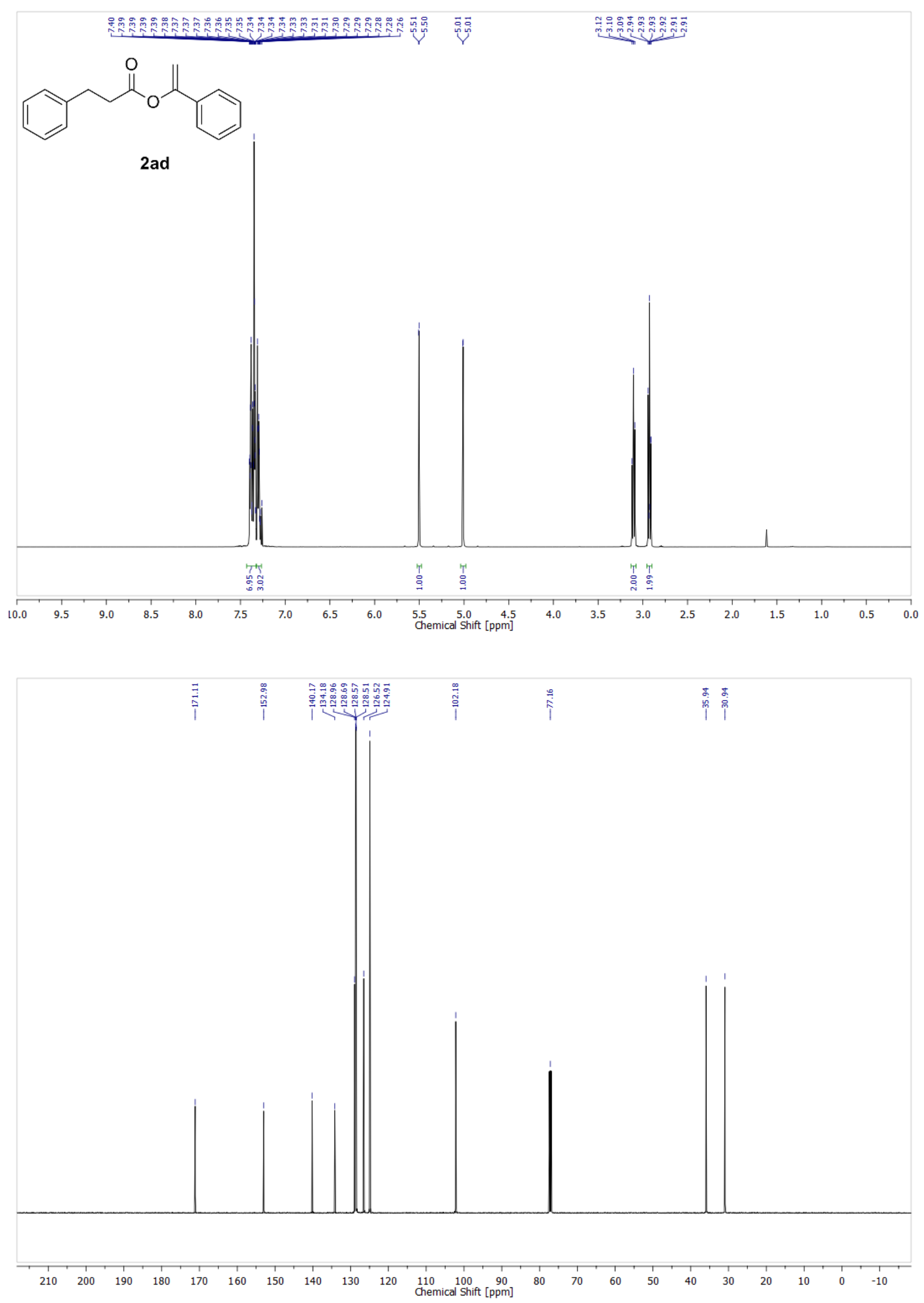

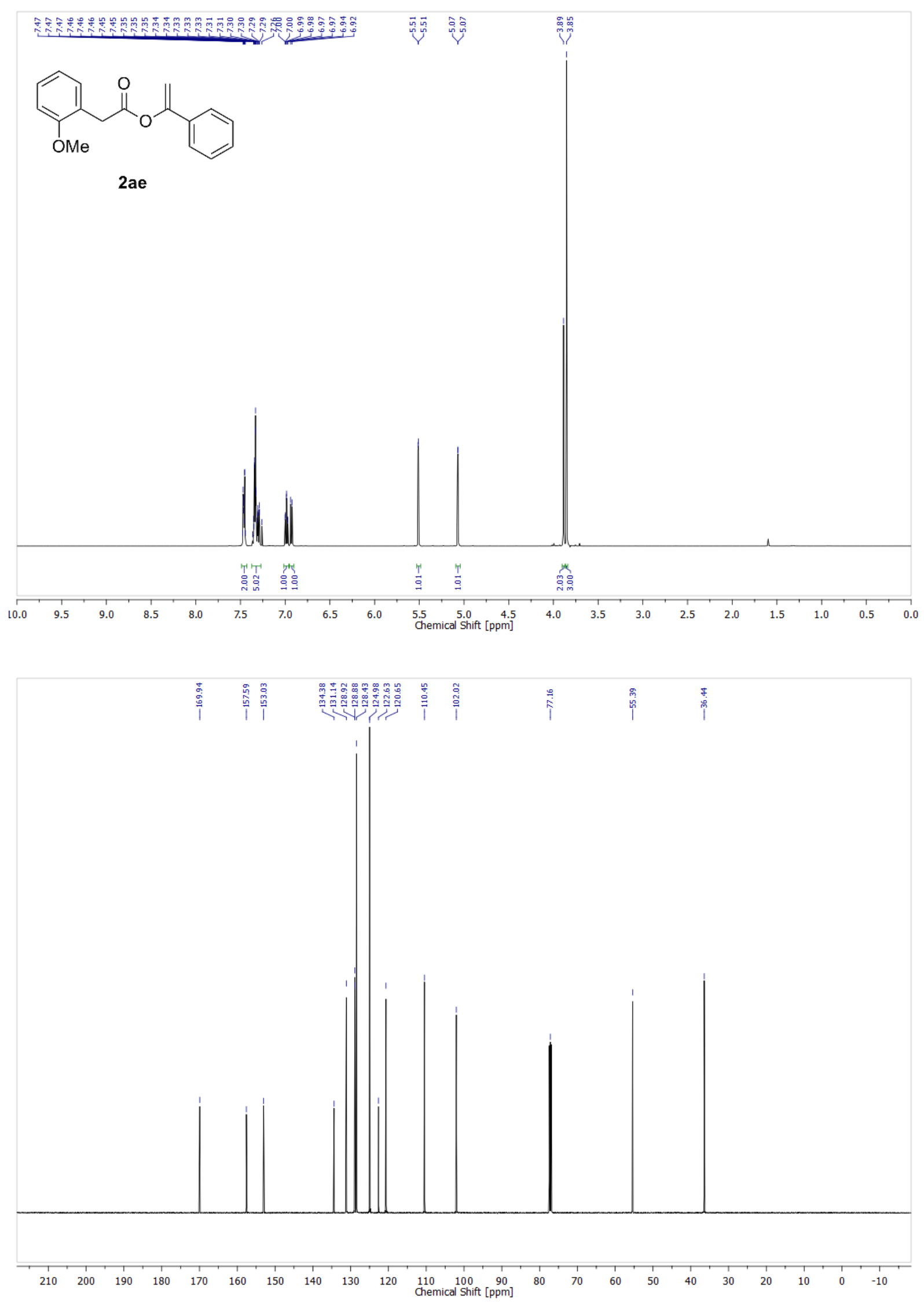

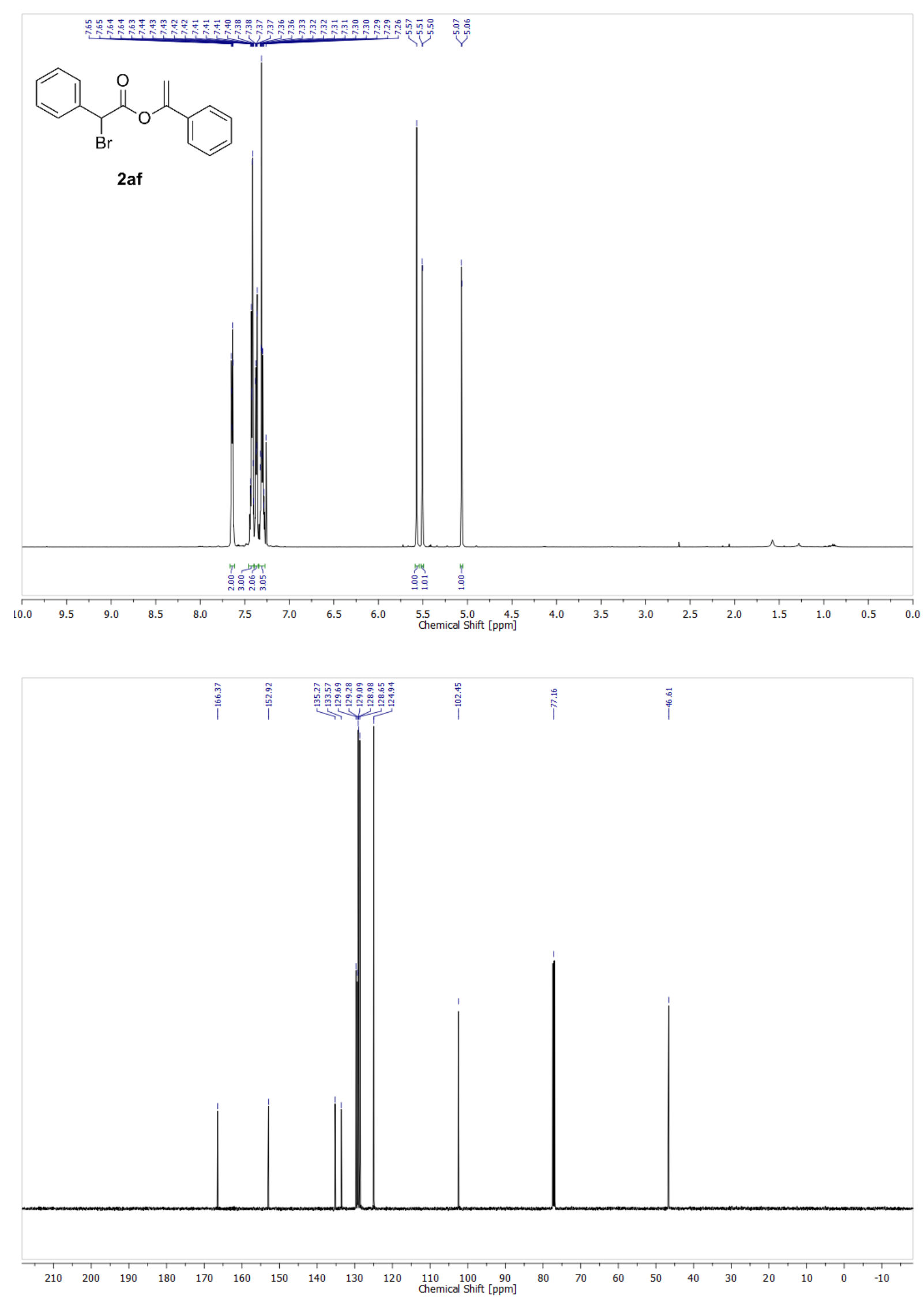

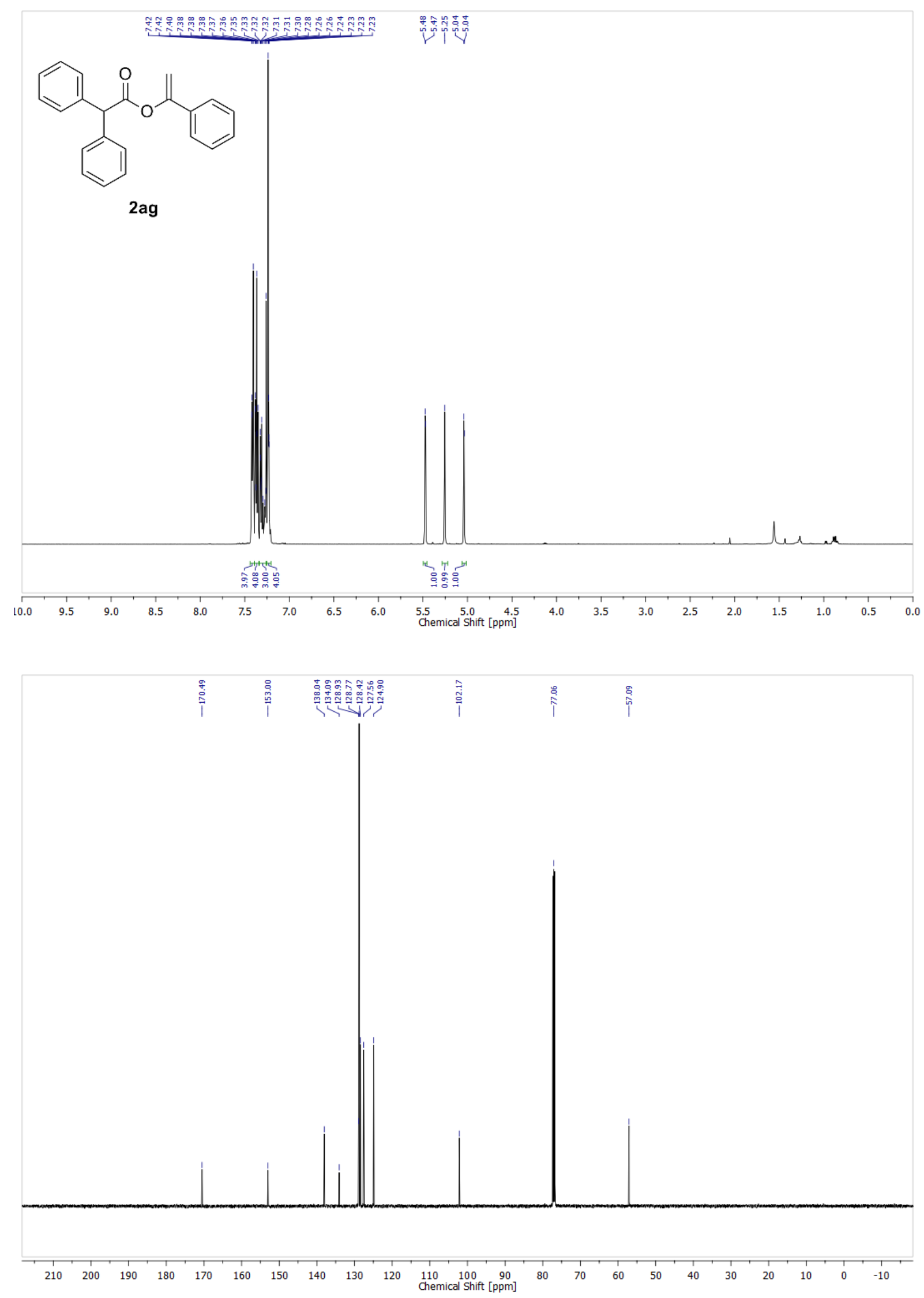

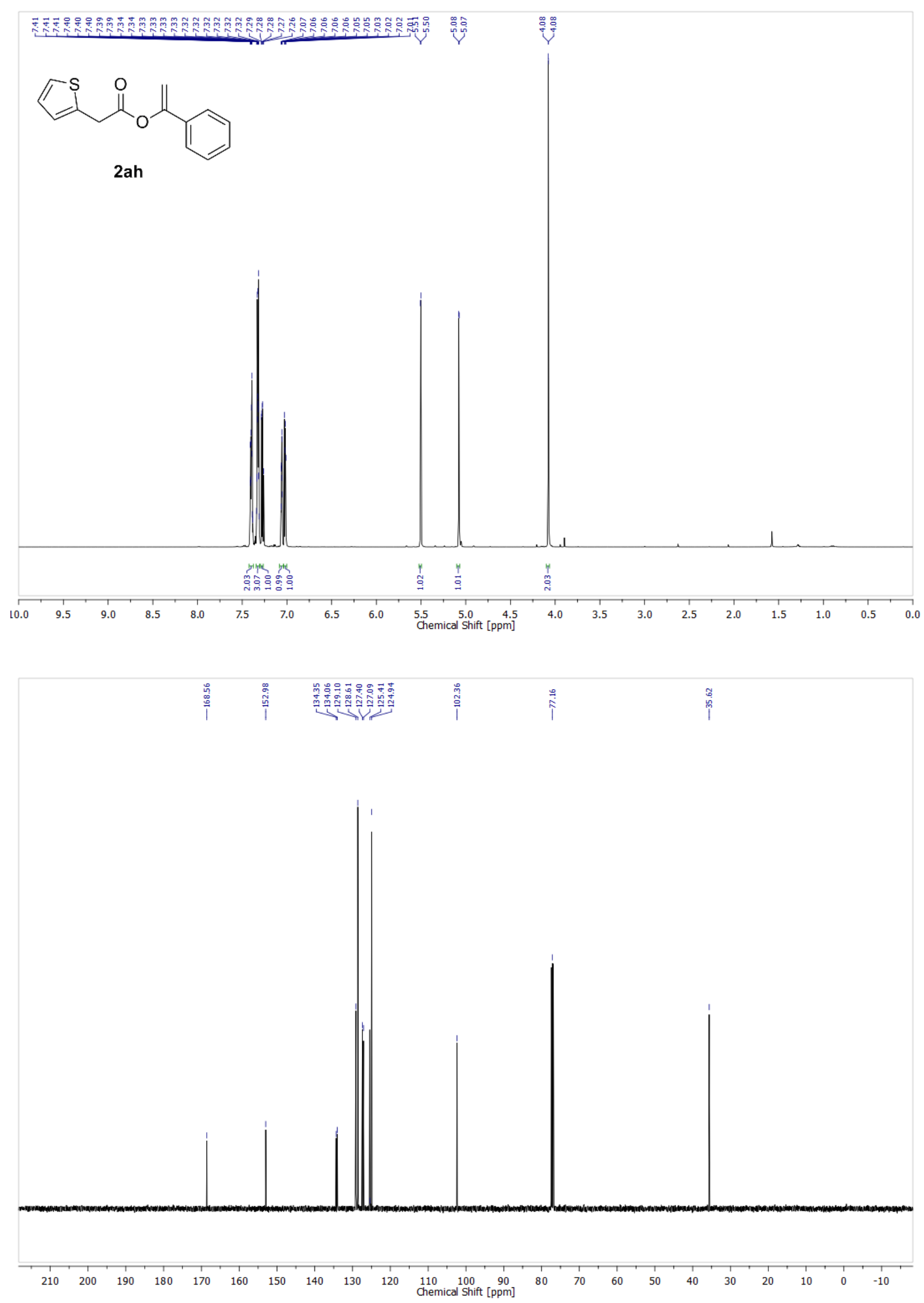

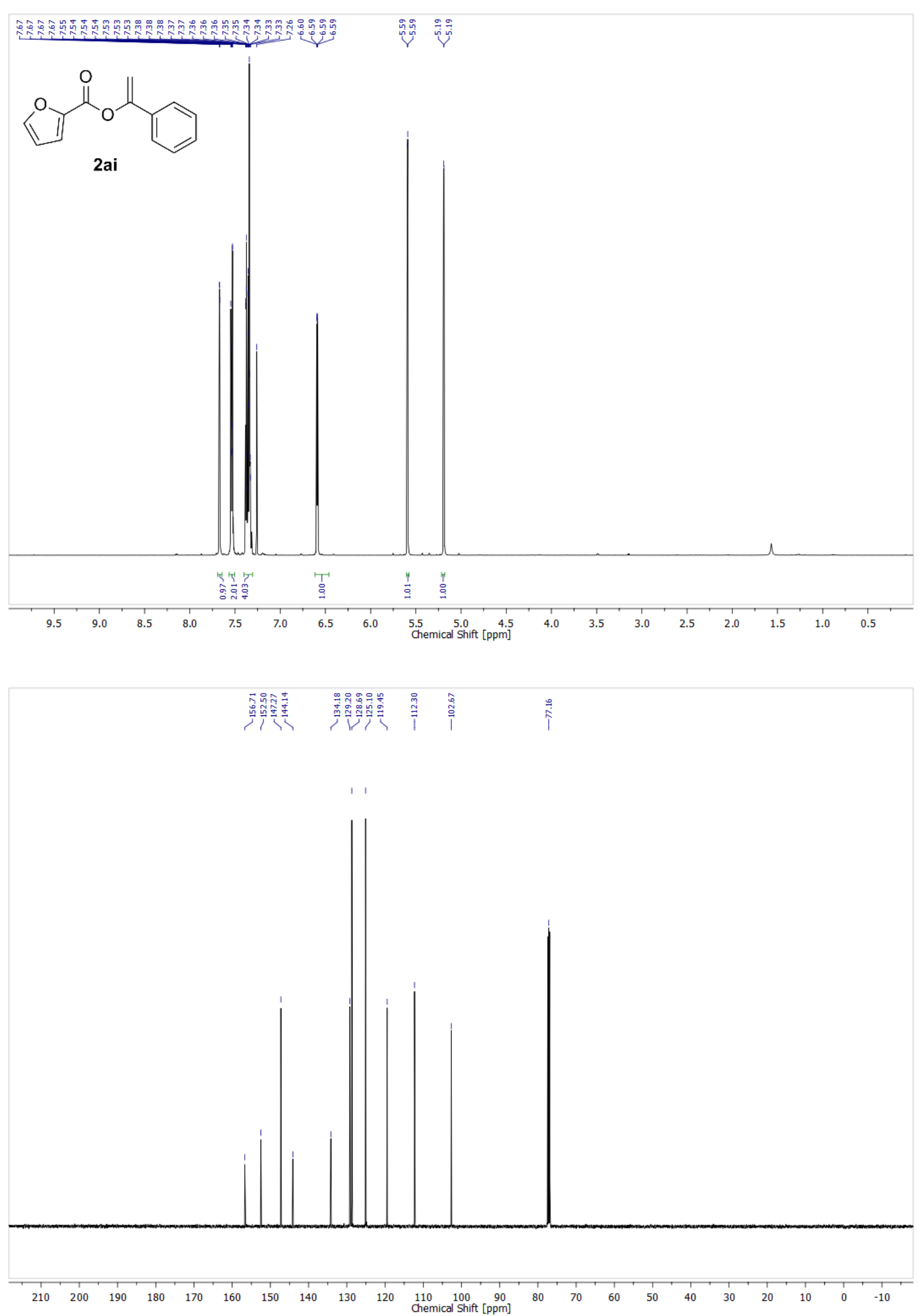

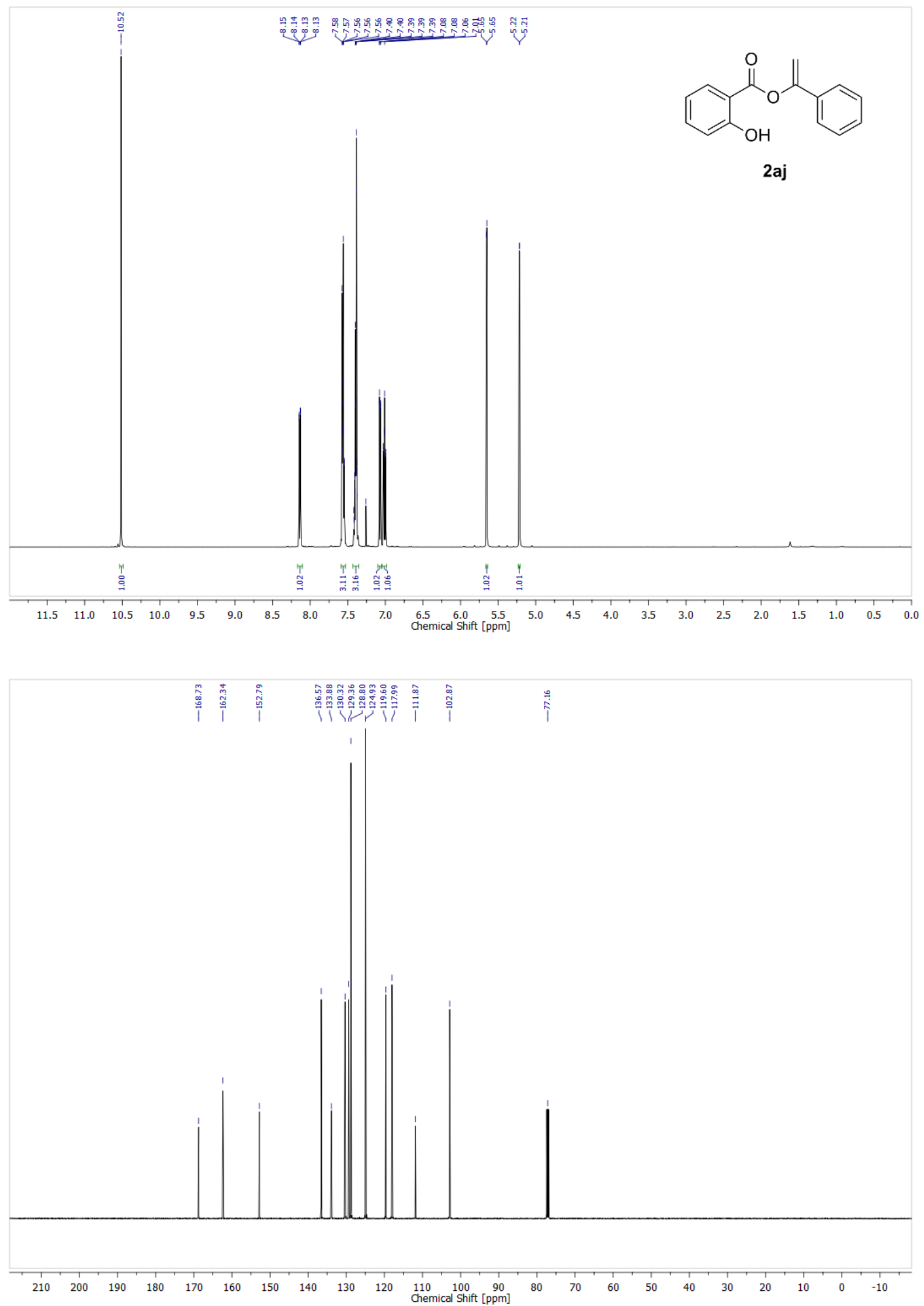

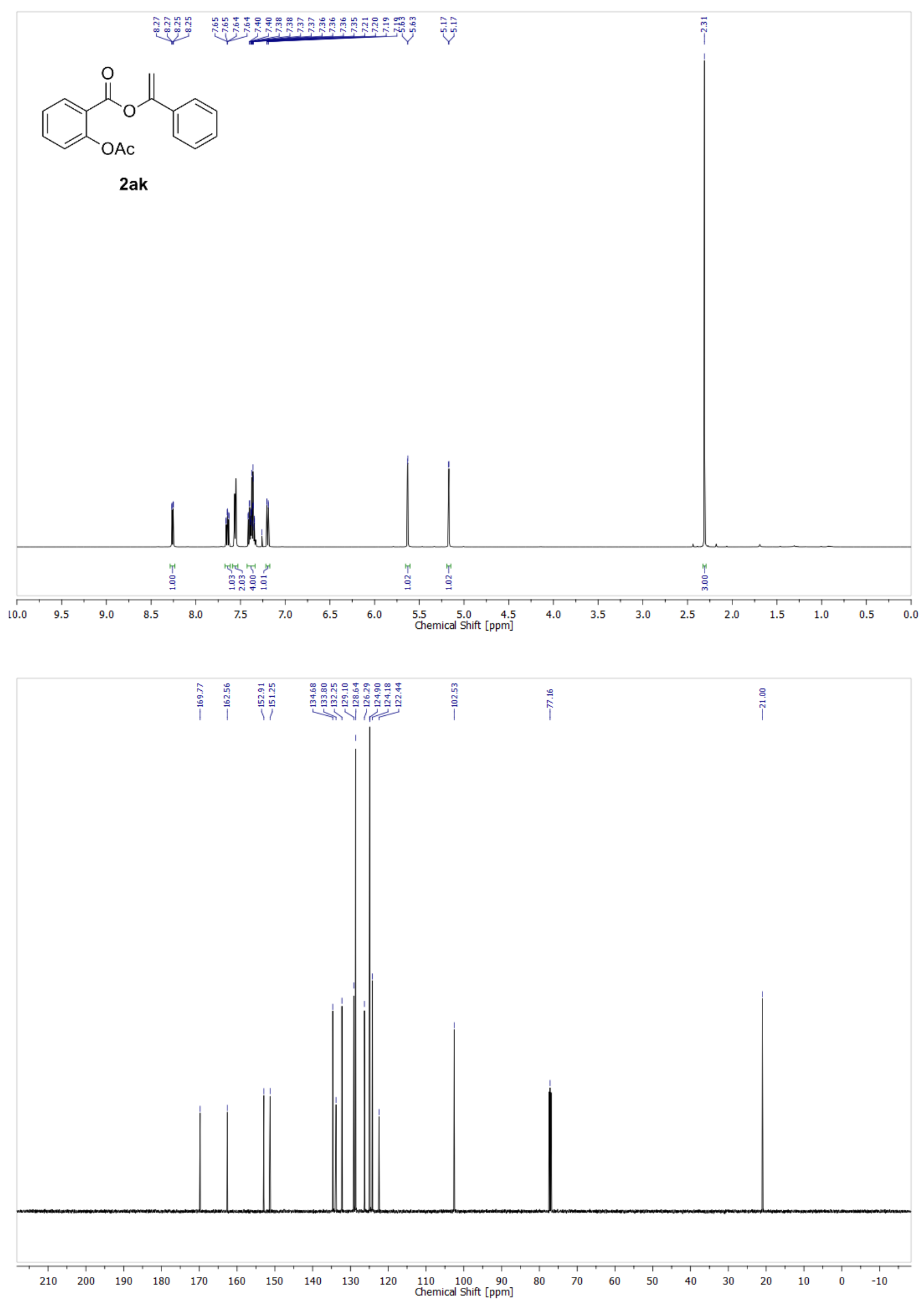

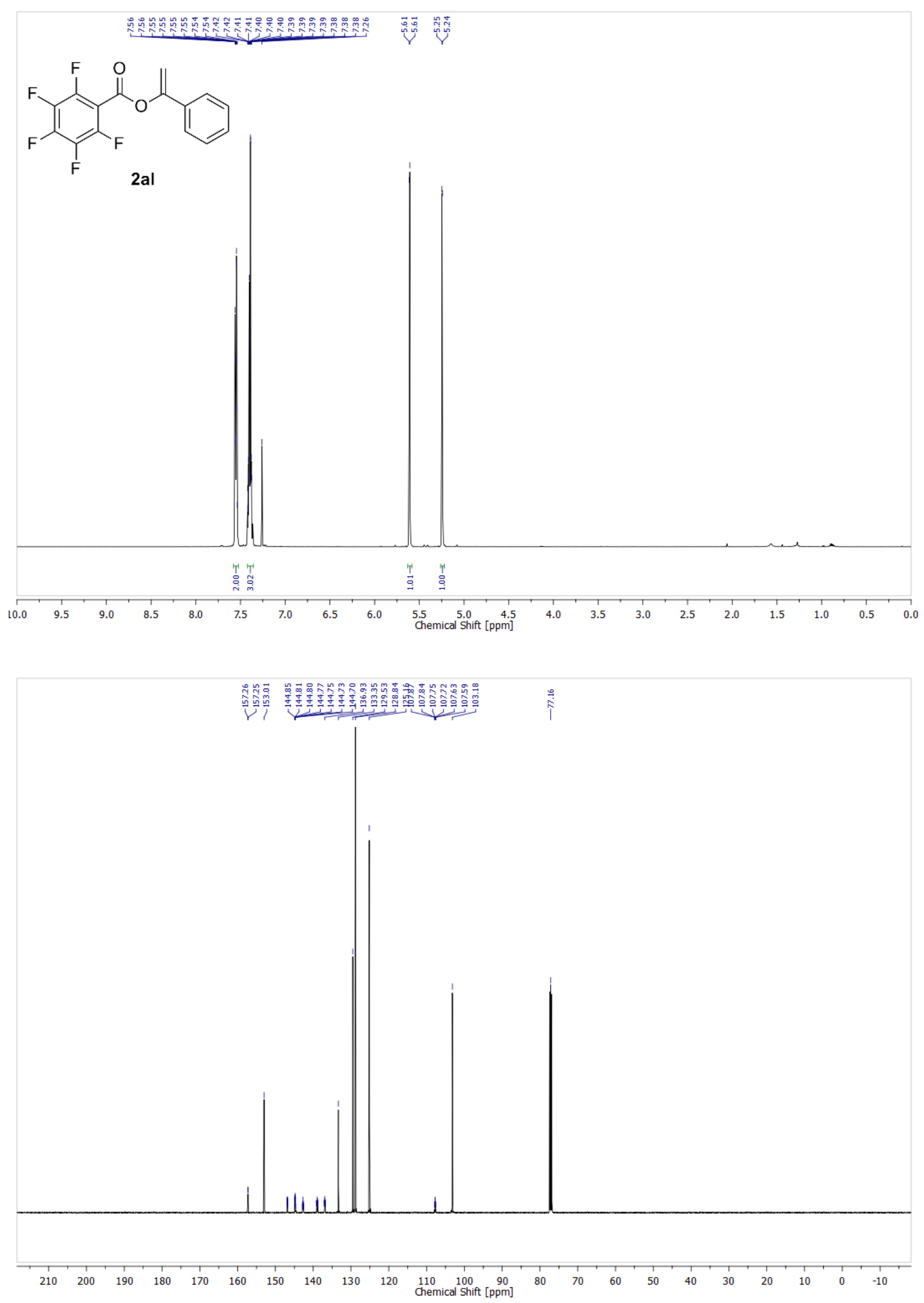

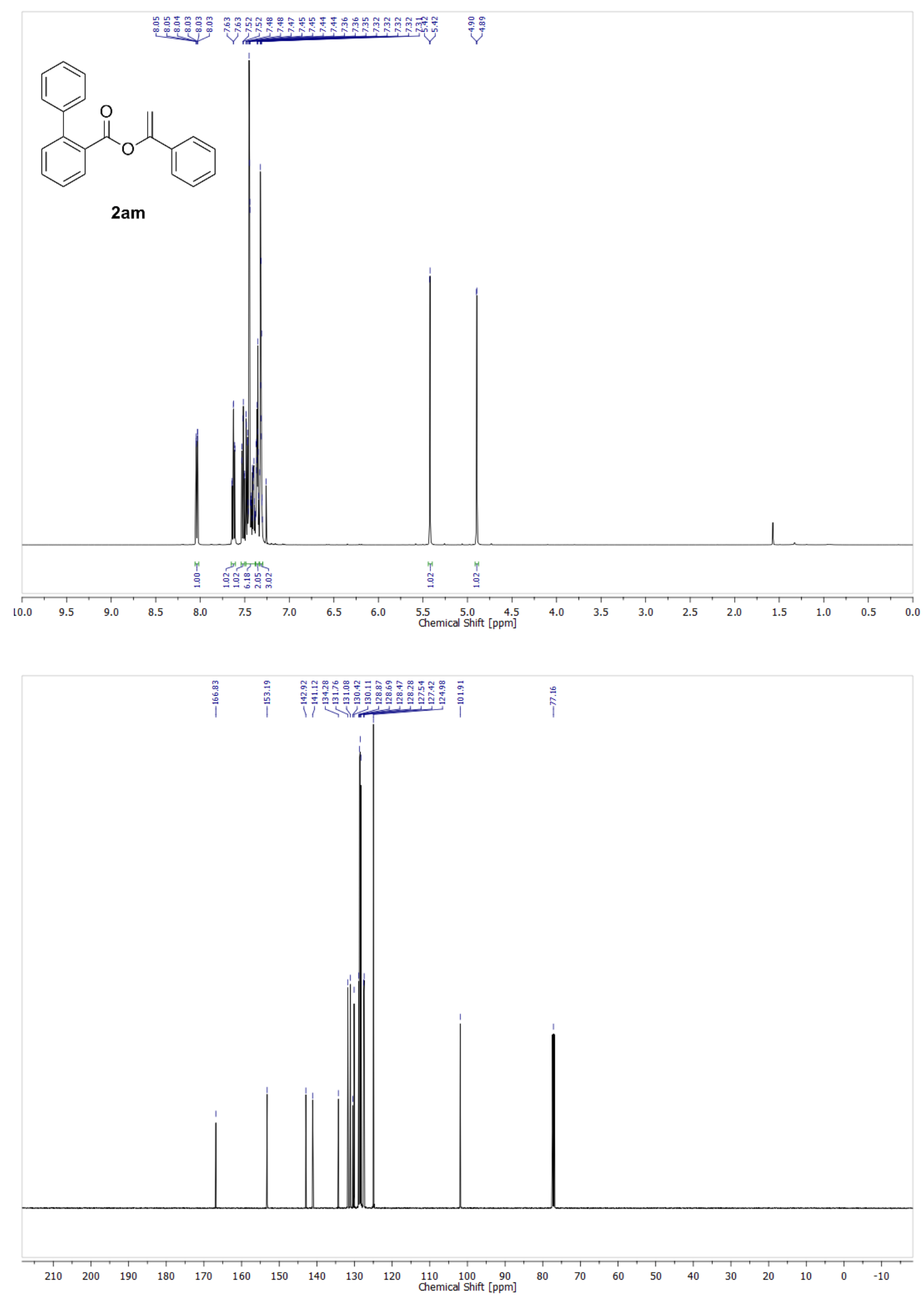

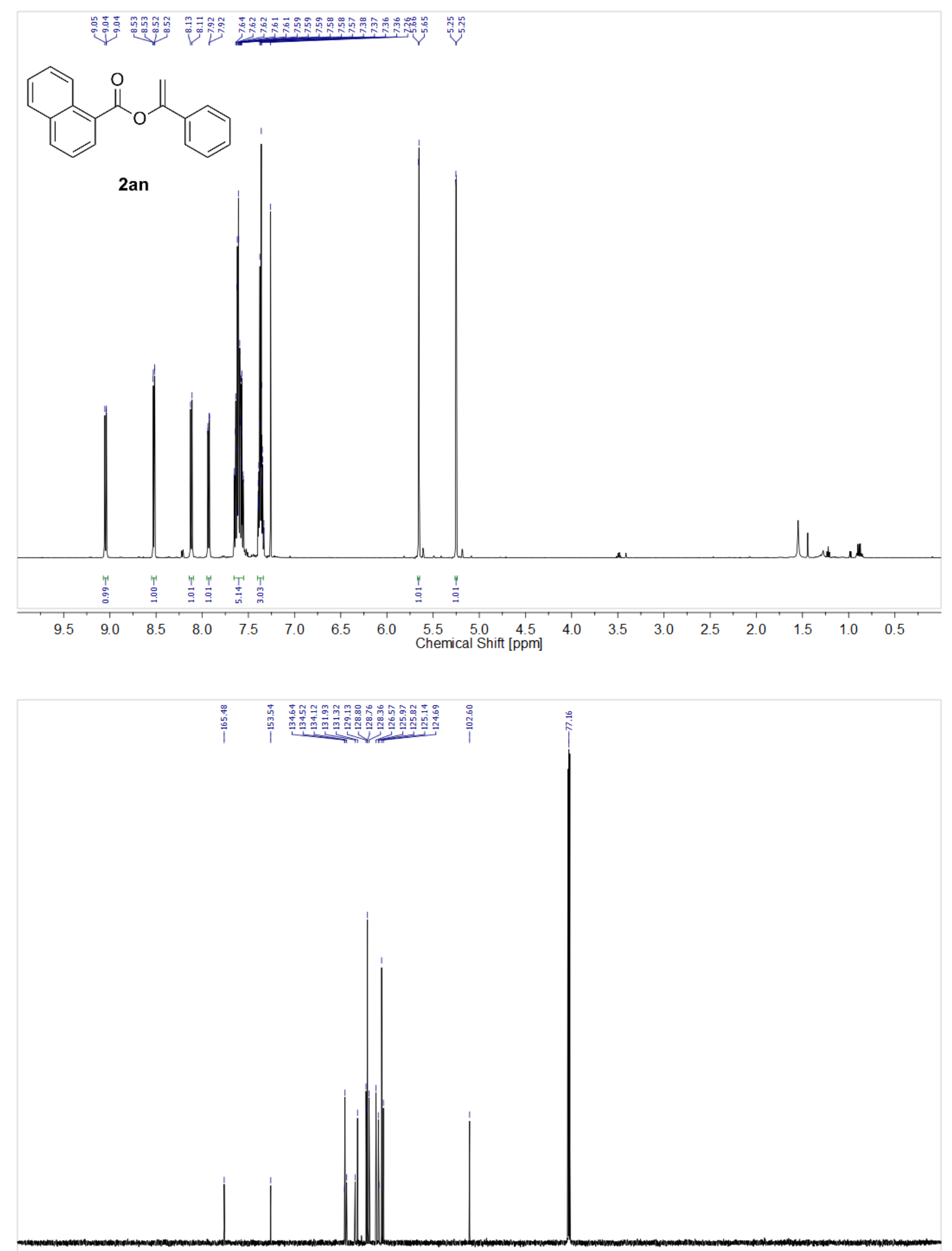

$\begin{array}{lllllllllllllllllllllllllllllllll}210 & 200 & 190 & 180 & 170 & 160 & 150 & 140 & 130 & 120 & 110 & 100 & 90 & 80 & 70 & 60 & 50 & 40 & 30 & 20 & 10 & 0 & -10\end{array}$ 

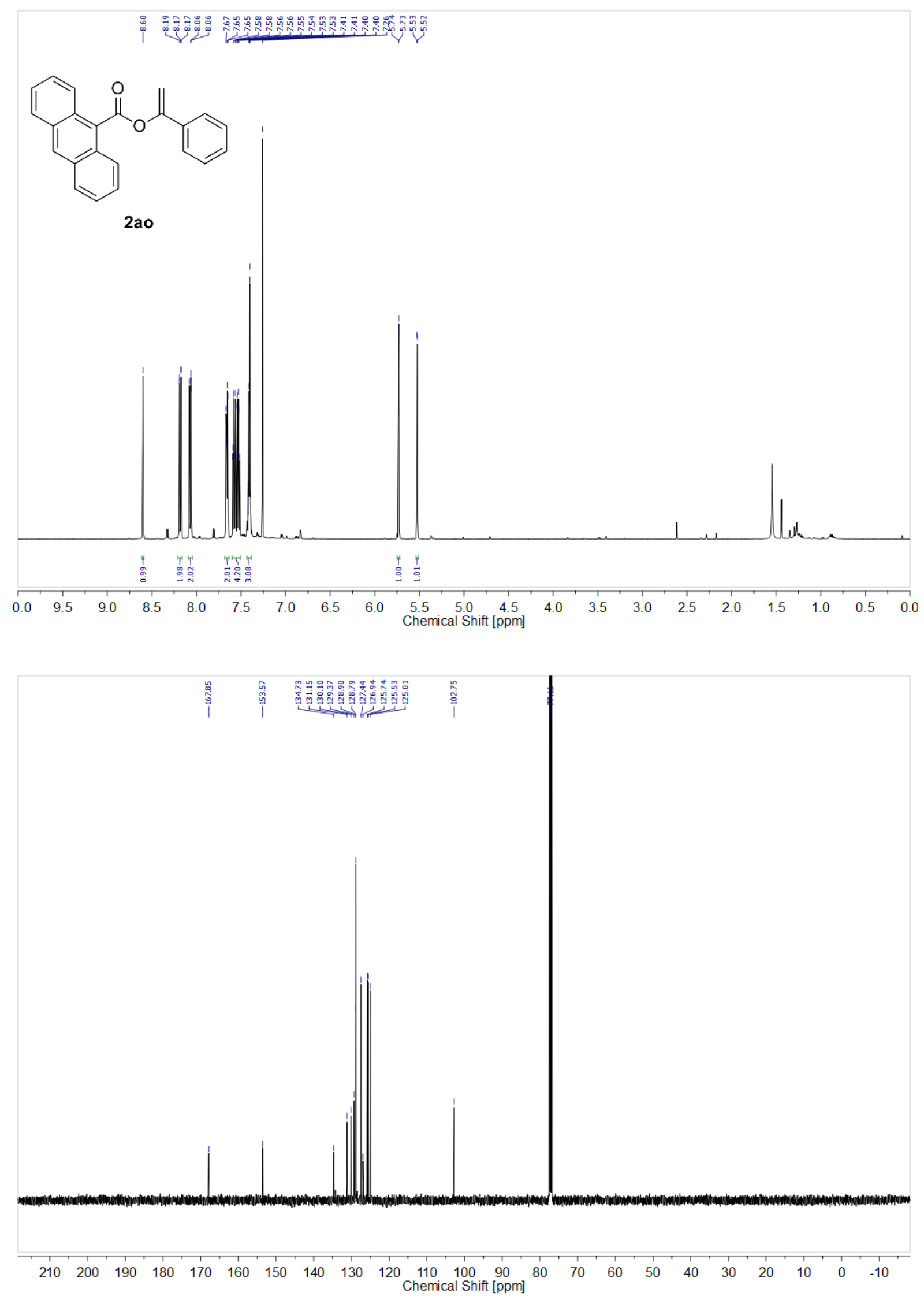
${ }^{1} \mathrm{H}$ and ${ }^{13} \mathrm{C}\left\{{ }^{1} \mathrm{H}\right\}$ NMR Spectra of Catalysis Product $2 \mathrm{bl}$ in $\mathrm{CDCl}_{3}$
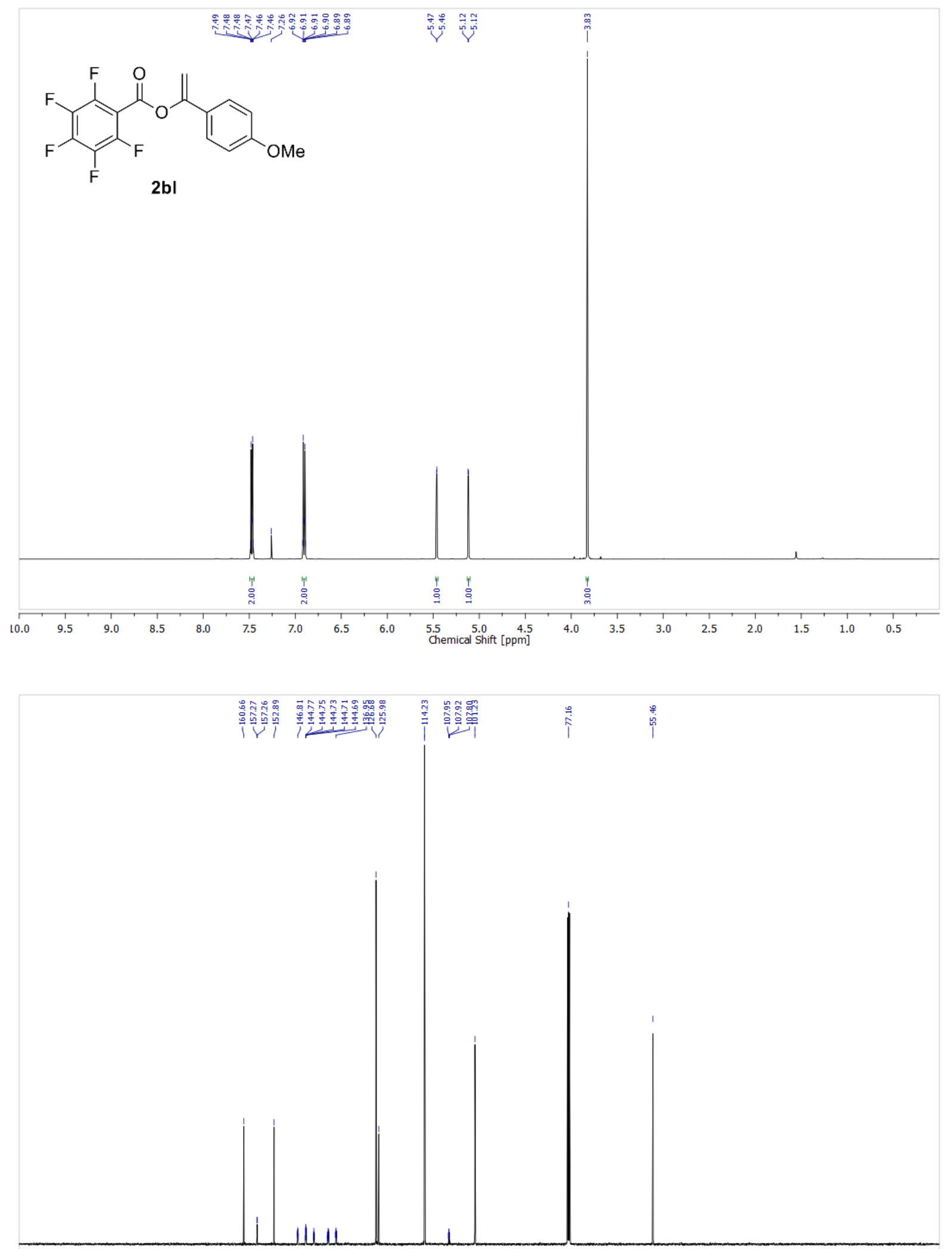

$\left.\begin{array}{llllllllllll}210 & 200 & 190 & 180 & 170 & 160 & 150 & 140 & 130 & 120 & 110 & 100 \\ \text { Chemical Shift }[\mathrm{ppm}\end{array}\right]$ 